\title{
The optical-to-radio spectral energy distributions of low-metallicity blue compact dwarf galaxies *
}

\author{
L. Hunt ${ }^{1}$, S. Bianchi ${ }^{1}$, and R. Maiolino ${ }^{2}$ \\ 1 INAF - Istituto di Radioastronomia/Sez. Firenze, Largo Enrico Fermi, 5, 50125 Firenze, Italy \\ e-mail: [hunt; sbianchi]@arcetri.astro.it \\ 2 INAF - Osservatorio Astrofisico di Arcetri, Largo Enrico Fermi, 5, 50125, Firenze, Italy \\ e-mail: maiolino@arcetri.astro.it
}

Received 11 October 2004 / Accepted 3 January 2005

\begin{abstract}
We present global spectral energy distributions (SEDs) from $0.3 \mu \mathrm{m}$ to $90 \mathrm{~cm}$ for a sample of seven low-metallicity blue compact dwarf galaxies (BCDs). In addition to data compiled from the literature, we report new SCUBA data for the galaxies in the sample, including the two most metal-poor star-forming galaxies known, IZw 18 and SBS 0335-052. The standard starburst templates, M 82 and Arp 220, do not give a good approximation to any of the BCD SEDs in our sample. Several SEDs are instead characterized by: (i) approximately flat radio spectra suggesting dominant thermal processes; (ii) farinfrared (FIR) spectra which peak at or shortward of $60 \mu \mathrm{m}$; (iii) a significant warm dust component and absence of Aromatic Features in Emission (or PAHs) in the mid-infrared (MIR). The SEDs of low-metallicity BCDs do not follow "canonical" scaling relations, and the "standard" star-formation indicators based on radio continuum, FIR, and MIR luminosities can be discrepant by factors of $\gtrsim 10$. We present new models of the dust SEDs from $5 \mu \mathrm{m}$ to $1 \mathrm{~cm}$, and derive infrared luminosities, dust distributions, temperatures, and masses. The observed SEDs and dust models are interpreted in terms of the active/passive regimes of star formation and trends with metallicity. Finally, we discuss the implications of our results on the $z \gtrsim 6$ starburst populations which will be detected by forthcoming submm and radio facilities.
\end{abstract}

Key words. galaxies: starburst - galaxies: ISM - ISM: dust, extinction - galaxies: star clusters - galaxies: evolution

\section{Introduction}

The physical processes which governed the formation of the first stars in the universe and the epoch during which it occurred are a main focus of modern cosmology. Measuring the cosmic star formation rate (SFR) of the universe as a function of redshift or look-back time has been the subject of much effort, initially at ultraviolet wavelengths (e.g., Madau et al. 1996), and more recently in the millimeter and radio regimes (e.g., Blain et al. 1999; Haarsma et al. 2000). Unlike the ultraviolet or optical spectral regions, emission at radio and (sub) millimeter wavelengths is unaffected by dust, which makes uncertain extinction corrections unnecessary. To exploit this advantage, emission at virtually every wavelength from the mid-infrared (MIR) to the radio has been used to trace star formation, both locally (Kennicutt 1998; Roussel et al. 2001; Condon et al. 1991) and at $z \lessgtr 4$ (Rowan-Robinson et al. 1997; Blain et al. 1999; Carilli \& Yun 1999; Chary \& Elbaz 2001; Garrett 2002; Yun \& Carilli 2002). Nevertheless, the conversion of MIR, farinfrared (FIR), sub-mm or radio continuum flux to a global SFR depends on canonical scaling relations (radio/FIR, MIR/FIR),

* Appendices A and B are only available in electronic form at http://www. edpsciences.org which may not be applicable to primordial star-forming systems at $z \geq 5$.

Even locally, there are some indications that SFR is not adequately traced by FIR or radio luminosity of low-luminosity or young star-forming galaxies (Klein et al. 1991; Roussel et al. 2003). Indeed, in dwarf galaxies, the scaling relations appear to hold only because both IR and radio emission underestimate the true SFR (Bell 2003). In these objects, particularly those of low metal abundance, the SFR estimated assuming the standard radio mix of thermal/non-thermal contributions can be underestimated by a factor of 5 or more (Kobulnicky \& Johnson 1999; Beck et al. 2002; Hunt et al. 2004). While rare in the nearby universe (see Kunth \& Östlin 2000), low-metallicity low-mass galaxies might be much more frequent at early times and high redshifts, given the predictions of the hierarchical merger models (e.g., Baugh et al. 1998; Cole et al. 2000). Indeed, such objects may represent the primordial "building blocks" - or "sub-galaxies" (Rees 1998) - in hierarchical scenarios of galaxy formation.

To better understand the effects of metallicity and luminosity on the properties of star-forming regions and investigate possible spectral templates for high- $z$ galaxies, we have undertaken a study of the spectral energy distributions 
Table 1. Sample galaxies.

\begin{tabular}{lccccccccc}
\hline \hline \multicolumn{1}{c}{ Name } & $a$ & $b$ & $z$ & $D(\mathrm{Mpc})^{a}$ & $M_{B}$ & $12+\log (\mathrm{O} / \mathrm{H})$ & $Z_{\odot} / Z^{b}$ & $\mathrm{~A}_{B}^{c}$ & Ref. $^{d}$ \\
\hline IZw 18 & 0.4 & 0.2 & 0.0025 & 13.0 & -14.1 & 7.19 & 55 & 0.138 & 1 \\
SBS 0335-052 & 0.2 & 0.2 & 0.0135 & 55.7 & -16.7 & 7.32 & 41 & 0.202 & 1 \\
II Zw 40 & 0.6 & 0.2 & 0.0026 & 12.1 & -14.9 & 8.09 & 7 & 3.538 & 2,3 \\
II Zw 70 = UGC 9560 & 0.7 & 0.3 & 0.0041 & 19.5 & -16.7 & 8.06 & 7 & 0.053 & 3 \\
NGC 5253 & 5.0 & 1.9 & 0.0014 & $4.1^{e}$ & -17.2 & 8.19 & 5 & 0.242 & $1,3,4$ \\
Mrk 33 = Haro 2 & 1.0 & 0.9 & 0.0049 & 23.4 & -18.4 & 8.40 & 3 & 0.052 & 5 \\
He 2-10 & 1.9 & 1.4 & 0.0029 & $9.0^{f}$ & -17.3 & 8.93 & 1 & 0.481 & 4 \\
\hline
\end{tabular}

${ }^{a}$ Distances calculated with $H_{0}=70 \mathrm{~km} \mathrm{~s}^{-1} \mathrm{Mpc}^{-1}$, and correcting to the CMB reference frame as described in the text. The distance for I Zw 18 derived by Östlin (2000) is $12.6 \mathrm{Mpc}$.

${ }^{b}$ Assuming solar $12+\log \mathrm{O} / \mathrm{H}=8.93$ (Anders \& Grevesse 1989).

${ }^{c}$ Galactic extinction $A_{B}$ (mag) taken from Schlegel et al. (1998).

${ }^{d}$ Reference for metallicity: (1) Izotov \& Thuan (1999); (2) Guseva et al. (2000); (3) Kobulnicky \& Skillman (1996); (4) Kobulnicky et al. (1999); (5) Legrand et al. (1997).

${ }^{e}$ Distance taken the Cepheid variable estimate given by Sandage et al. (1994).

${ }^{f}$ Distance taken from Kobulnicky \& Johnson (1999).

(SEDs) of low-metallicity blue compact dwarfs (BCDs). Lowmetallicity galaxies are in fact the most viable local laboratories with which to study primordial galaxy formation, because of their low mass and chemically unenriched interstellar medium (ISM). However, such objects cannot be compared to the massive "SCUBA galaxies" and Lyman-break systems which are already chemically enriched at $z \lesssim 3$ (Pettini et al. 2001; Tecza et al. 2004; Shapley et al. 2004). At higher redshifts $z \gtrsim 6$, truly primordial star formation must occur in very low or zero metallicity environments, and metal-poor BCDs are the only way to study such processes locally. Because IR-radio correlations are used to infer SFR for galaxies observed at high redshift, it is important to verify that all galaxies, even the most chemically unevolved, follow such correlations. Moreover, dust grain properties may depend on metallicity and on the age of the stellar populations. Stellar ages at cosmological distances have a strong bearing on the origin of the dust (stellar winds of evolved stars versus supernovae).

In this paper, we present global fluxes from $0.3 \mu \mathrm{m}$ to $90 \mathrm{~cm}$ for $7 \mathrm{BCDs}$ with metallicities ranging from $1 / 50 Z_{\odot}$ to solar. In addition to our new near-infrared (NIR) images, we have also acquired data from the HST and JCMT/SCUBA archives, and gleaned optical, infrared, and radio data from the literature. In Sect. 2, we describe the sample, the data, and the data reduction. Section 3 presents the SEDs for the sample BCDs, and Sect. 4 discusses their interpretation in terms of "active" and "passive" star formation. New SED models for the BCDs in our sample with sufficient data are given in Sect. 5, and scaling laws among mid-, far-infrared, and radio emission are described in Sect. 6. We discuss the implications of our results on star-formation history and metallicity in Sects. 7 and 8, and in the context of high-redshift star formation in Sect. 9. If lowmetallicity BCD SEDs differ from the usual templates (e.g., M 82 and Arp 220), it will be necessary to reassess the interpretation of the faint sources at $z \gtrsim 6$ expected to be detected with the new sub-mm, mm, and radio facilities such as APEX, ALMA, SKA, and LOFAR.

\section{The sample and the data}

Our sample is based on all the low-metallicity BCDs available from the JCMT/SCUBA archive as of December 2003. This resulted in seven BCDs with metallicities ranging from solar to $\lesssim 1 / 50 Z_{\odot}$. Our sample includes the two most metalpoor star-forming galaxies known, IZw $18\left(\lessgtr 1 / 50 Z_{\odot}\right)$ and SBS 0335-052 $\left(1 / 41 Z_{\odot}\right)$. Table 1 lists the basic properties of the sample. Properties are taken from NED, with the exception of the metallicities, which were gleaned from the references listed in the last column of the table. Distances were calculated assuming a Hubble constant $H_{0}=70 \mathrm{~km} \mathrm{~s}^{-1} \mathrm{Mpc}^{-1}$, after correcting the heliocentric radial velocities to the CMB reference frame according to the prescription of the Third Reference Catalogue (RC3, de Vaucouleurs et al. 1991). While this sample is neither complete nor homogeneously selected, it contains many heavily studied BCDs and enables a comparison of their spectral properties. It also has the virtue of spanning a factor of 50 in metallicity, and a factor of $\gtrsim 10^{4}$ in blue luminosity. With the exception of Mrk 33, all the sample galaxies are BCDs according to the criteria (luminosity, central color, peak surface brightness) set out by Gil de Paz et al. (2003). Mrk 33, with an absolute $B$ magnitude of -18.4 , is slightly too luminous to be considered a true dwarf galaxy.

\subsection{SCUBA data reduction}

Data reduction of SCUBA data was performed in the standard way, using the dedicated software SURF by Jenness \& Lightfoot (1998). The data were first flat-fielded to take into account different bolometer sensitivities, then corrected for atmospheric extinction. Atmospheric opacities were mainly derived from the $225 \mathrm{GHz}$ opacity monitor, using the well studied relations between the optical depth at $225 \mathrm{GHz}$ and those at $450 \mu \mathrm{m}$ and $850 \mu \mathrm{m}$. Bolometers that appeared free of source emission were used to remove residual sky noise fluctuations. 
Table 2. Total SCUBA fluxes for sample galaxies.

\begin{tabular}{lcrlr}
\hline \hline \multicolumn{1}{c}{ Name } & $\begin{array}{c}450 \mu \mathrm{m} \\
(\mathrm{mJy})\end{array}$ & $\begin{array}{r}850 \mu \mathrm{m} \\
(\mathrm{mJy})\end{array}$ & Comments \\
\hline IZw 18 & - & $<2.5$ & \\
II Zw 40 & $400 \pm 90$ & $90 \pm 10$ & Resolved at $850 \mu \mathrm{m}$ \\
SBS 0335-052 & - & $<5$ & \\
II Zw 70 = UGC 9560 & - & $<20$ & \\
NGC 5253 & $<2200$ & $180 \pm 20$ & Resolved at $850 \mu \mathrm{m}$ \\
He 2-10 & $580 \pm 150$ & $140 \pm 15$ & Resolved at $850 \mu \mathrm{m}$ \\
Mrk 33 = Haro 2 & $<170$ & $50 \pm 10$ & Resolved at $850 \mu \mathrm{m}$ \\
\hline
\end{tabular}

Flux calibration was achieved from observations of secondary calibrators or Uranus, when available.

Five objects were observed in jiggle-map mode, with data available for both the short $(450 \mu \mathrm{m})$ and long $(850 \mu \mathrm{m})$ wavelength arrays. Maps were produced after rebinning the data in the RA-Dec plane. Total fluxes were measured integrating the source signal (and the calibrator) over a selected aperture, as described in Dunne et al. (2000) and Jenness et al. (2002). For detected sources, apertures were selected to enclose regions where $S / N>2$ (unless they were smaller than a 40" circular aperture, which was used in these cases). II Zw 40 was observed in 10 nights between February 2000 and January 2001, for a total number of 315 integrations (10 integrations require about $20 \mathrm{~min}$ to be completed, with the bolometer being onsource half of the time). The sky opacity at $850 \mu \mathrm{m}$ was in the range $\tau_{850}=0.2-0.3$. The object was detected both at $450 \mu \mathrm{m}$ and $850 \mu \mathrm{m}$. At the longer wavelength the galaxy is well resolved by SCUBA $\left(F W H M \approx 15^{\prime \prime}\right)$. He 2-10 was observed in two nights in December 2000 (95 integrations) with sky opacity $\tau_{850}=0.2-0.3$. The object has also been detected at both wavelengths. II Zw 70 and NGC 5253 were observed together in a single night (1999, January 13) with $\tau_{850}=0.3-0.4$ (20 integrations each). Only NGC 5253 was detected, at $850 \mu \mathrm{m}$. Finally, Mrk 33 was observed during several nights between March 1998 and January 1999 (106 integrations), with $\tau_{850}$ in the range $0.1-0.5$. It also has been detected at $850 \mu \mathrm{m}$. Fluxes for these objects (and 2- $\sigma$ upper limits) are reported in Table 2. To our knowledge, the data for only two of these objects have been previously published (namely NGC 5253 and Mrk 33: James et al. 2002); the fluxes from the literature are in excellent agreement with our independent data reduction, the results of which are reported in Table 2.

The two remaining objects of our sample, IZw 18 and SBS 0335-052 have been observed in photometry mode in February 2000, in nights with optical depths $\tau_{850}=0.3-0.4$. A total number of 497 integrations were spent on IZw 18 and 200 on SBS 0335-052. Neither object was detected; upper limits are given in Table 2.

\subsection{Data from the literature}

We searched the literature for optical, NIR, mid- and farinfrared, sub-mm, mm, and radio data. Data from our own observations were incorporated for Mrk 33 (Haro 2), II Zw 40, and
SBS 0335-052. Because we aim in this paper to derive the SED for the entire galaxy, rather than individual regions, we have placed greater emphasis on the largest aperture available and total fluxes. This is a problem particularly for the older midinfrared (MIR) observations with photometers, which were, of necessity, performed with small apertures. Global fluxes are frequently unavailable, and comparison of different aperture sizes at different wavelengths is the major source of the uncertainties in our SEDs.

For wavelengths $<5 \mu \mathrm{m}$, Galactic extinction was corrected according to the values given by Schlegel et al. (1998) (see Table 1), and using the Galactic extinction curve with $R_{V}=3.1$ by Cardelli et al. (1989). Corrected optical magnitudes were converted to fluxes using the zero points by Bessell (1979), and NIR/MIR ones given by the UKIRT web page ${ }^{1}$. The UKIRT web page is based on the calibrations given by Beckwith et al. (1976) and Tokunaga (1984). Interpolation was applied as necessary.

Fluxes from the Infrared Astronomical Satellite (IRAS) were taken either from the NASA/IPAC Extragalactic Database (NED), or from published papers. Although data were examined on a case-by-case basis, single-dish radio observations were generally preferred to high-resolution VLA data, because of the possibility of losing flux from low spatial frequency filtering.

\subsection{ISO data for SBS 0335-052}

To re-evaluate the $65 \mu \mathrm{m}$ flux for SBS 0335-052, in light of the Spitzer ${ }^{2}$ results (Houck et al. 2004), we extracted from the ISO archive the ISOPHOT data for this object. The pipeline reduction and calibration was used, and aperture photometry was performed with IRAF ${ }^{3}$. We obtain a weak $(\sim 2 \sigma)$ detection for SBS $0335-052$ at $65 \mu \mathrm{m}$ of $\sim 44 \mathrm{mJy}$. This is substantially lower than the 112 mJy estimate by Plante \& Sauvage (2002), but in much better agreement with the Spitzer IRS spectrum reported by Houck et al. (2004).

\subsection{NIR data for Mrk 33 and II Zw 40}

For two BCDs in our sample, we present new NIR imaging data. $J(1.2 \mu \mathrm{m}), H(1.6 \mu \mathrm{m})$, and $K(2.2 \mu \mathrm{m})$ images of II Zw 40 were acquired with the 3.8-m United Kingdom Infrared Telescope $\left(\mathrm{UKIRT}^{4}\right.$ ) equipped with IRCAM3. The observations are part of our ongoing project of NIR imaging and spectroscopy of BCDs (see Hunt et al. 2003). The IRCAM3 plate scale is 0 '. 28 per pixel, with a total field-of-view

\footnotetext{
1 wwW.jach.hawaii.edu/JACpublic/UKIRT/astronomy/ conver.html

2 The Spitzer Space Telescope is a NASA mission managed by the Jet Propulsion Laboratory. See www . spitzer . caltech. edu.

3 IRAF is the Image Analysis and Reduction Facility made available to the astronomical community by the National Optical Astronomy Observatory, which is operated by AURA, Inc., under contract with the US National Science Foundation.

4 The United Kingdom Infrared Telescope is operated by the Joint Astronomy Centre on behalf of the UK Particle Physics and Astronomy Research Council.
} 
(FOV) of $72^{\prime \prime} \times 72^{\prime \prime}$. We acquired $J, H$, and $K$ images of Mrk33 with ARNICA mounted on the 1.5-m Italian Telescope at Gornergrat $\left(\mathrm{TIRGO}^{5}\right)$. The ARNICA plate scale is $0{ }^{\prime} 97$ per pixel with a FOV of $4.1^{\prime} \times 4.1^{\prime}$. Both cameras house NICMOS3 $256^{2}$ detectors.

Each galaxy was observed by alternating frames on the source and on adjacent empty sky positions, beginning and ending each observing sequence with a sky position. For the UKIRT data, before the beginning of each sequence, dark exposures were acquired with the same parameters as the subsequent science frames. Individual frames were dark-subtracted (in the case of IRCAM3) and flat-fielded with the average of adjacent empty sky frames, after editing them for stars (to avoid "holes" in the reduced frames) and applying an average-sigma clipping algorithm. The reduced frames were then aligned and averaged. All data reduction was carried out in the IRAF environment.

Photometric calibration at both telescopes was performed by observing standard stars from the UKIRT Faint Standard List (Hawarden et al. 2001) before and after the source observations. Each standard star was measured in several different positions on the array, and flat-fielded by dividing the clipped mean of the remaining frames in the sequence. To correct the standard-star photometry for atmospheric extinction, we used the mean extinction coefficients given by each observatory. Virtual aperture photometry was performed for both objects, but Mrk 33 is sufficiently large that we opted for asymptotic magnitudes derived from growth curves, rather than truncating to a given aperture. The magnitudes derived in this way agree to within $0.1 \mathrm{mag}$ to the total magnitudes given by Jarrett et al. (2003). Galactic extinction was corrected for and conversion to flux was achieved as described in Sect. 2.2.

\section{Spectral energy distributions}

The SEDs of our sample galaxies are presented graphically in Figs. 1 and 2. The data are given in the Appendix as separate tables, together with the references. Although we call them "SEDs", we have plotted here the flux (mJy) versus wavelength, a usual, but conceptually unsound, convention. We have done so because it is much easier to intuitively interpret the trends in the distributions, and identify low flux levels because of the vertical scaling common to all graphs.

We have compared the SEDs shown in Figs. 1 and 2 with the spectrophotometric models for M 82 and Arp 220 given by Silva et al. (1998) and Bressan et al. (2002). These two galaxies were chosen because they are frequently used as templates for photometric redshift determinations (e.g., Hughes et al. 2002). The models have been normalized by calculating the mean offset over the entire ensemble of data points in logarithmic flux units. Such a procedure is equivalent to adjusting the vertical scale so as to minimize the residuals data-model. The global spectral normalization is crucial to interpret deviations from the models because of the insidious effects of dust extinction, radio free-free absorption, and different dust temperatures.

\footnotetext{
5 TIRGO (Gornergrat, $\mathrm{CH}$ ) is operated by the Istituto di Radioastronomia-Sezione Firenze.
}

Problems with total versus large-aperture flux are apparent in NGC 5253 and He 2-10 (Fig. 1). In both cases, excluding the lower-flux points does not change the normalization relative to the models; we have shown all data and included them in the best-fit assessment (see below).

\subsection{Deviations from the standard starburst templates}

To assess how well the SEDs of M 82 and Arp 220 fit the lowmetallicity BCDs in our sample, we calculated the average rms (logarithmic) residuals after normalization. We also divided the SED into four spectral regions, and calculated the average mean offset (normalization) and rms residuals individually for each region: optical+NIR $(\lambda \leq 4 \mu \mathrm{m})$, MIR $(4<\lambda \leq 30 \mu \mathrm{m})$, FIR-mm $(30<\lambda \leq 7000 \mu \mathrm{m})$, and radio $(\lambda \geq 7.0 \mathrm{~mm})$. The models were interpolated to the wavelength of the observation. In every case, the model which gave the smallest rms residuals after normalization is M 82; Arp 220 is not a good approximation to any of the galaxies in our sample.

The results of the normalization/RMS procedure are given in Table 3 for the best-fit model (M 82). Column 2 gives the (logarithmic) rms residuals over the entire SED, and Cols. 3 through 6 contain first the mean offset, and then the rms residual for the individual spectral regions. In all columns, the number enclosed in parentheses is the number of data points. The normalization procedure guarantees that the global mean offset is zero (see previous section). All units in the table are logarithmic. Figure 3 shows the deviations from the M 82 SED graphically.

Inspection of Figs. 1, 2, and Table 3 reveals several important features.

Radio: three of the BCDs (IIZw 40, NGC 5253, SBS 0335-052) have approximately flat global radio emission; on galaxy scales the radio emission appears to be dominated by thermal processes.

FIR-mm: in three objects (II Zw 40, NGC 5253, He 2-10), the $60-100 \mu \mathrm{m}$ emission is significantly larger than the bestfit template. In SBS $0335-052$, the $65 \mu \mathrm{m}$ point falls well below it.

MIR: in the objects for which we have considerable spectral coverage at the mid-infrared wavelengths (II Zw 40, NGC 5253, SBS 0335-052), the observed MIR spectrum is not well fit by the M 82 template. First, the flux for $\lambda \gtrsim$ $12 \mu \mathrm{m}$ exceeds the template significantly. Second, there are no PAH features (or Aromatic Features in Emission, AFEs) in these objects, unlike M 82 (and Arp 220).

Optical+NIR: three BCDs (II Zw 40, IZw 18, SBS 0335-052) have optical+NIR emission which falls well below the M 82 template. II Zw 70 appears to have an optical excess relative to $\mathrm{M} 82$, but a NIR deficit.

\section{Active and passive star formation}

The preceding section suggests that three galaxies deviate from the M 82 SED over the entire spectral range covered by our SEDs: II Zw 40, NGC 5253, SBS 0335-052. IZw 18 differs 


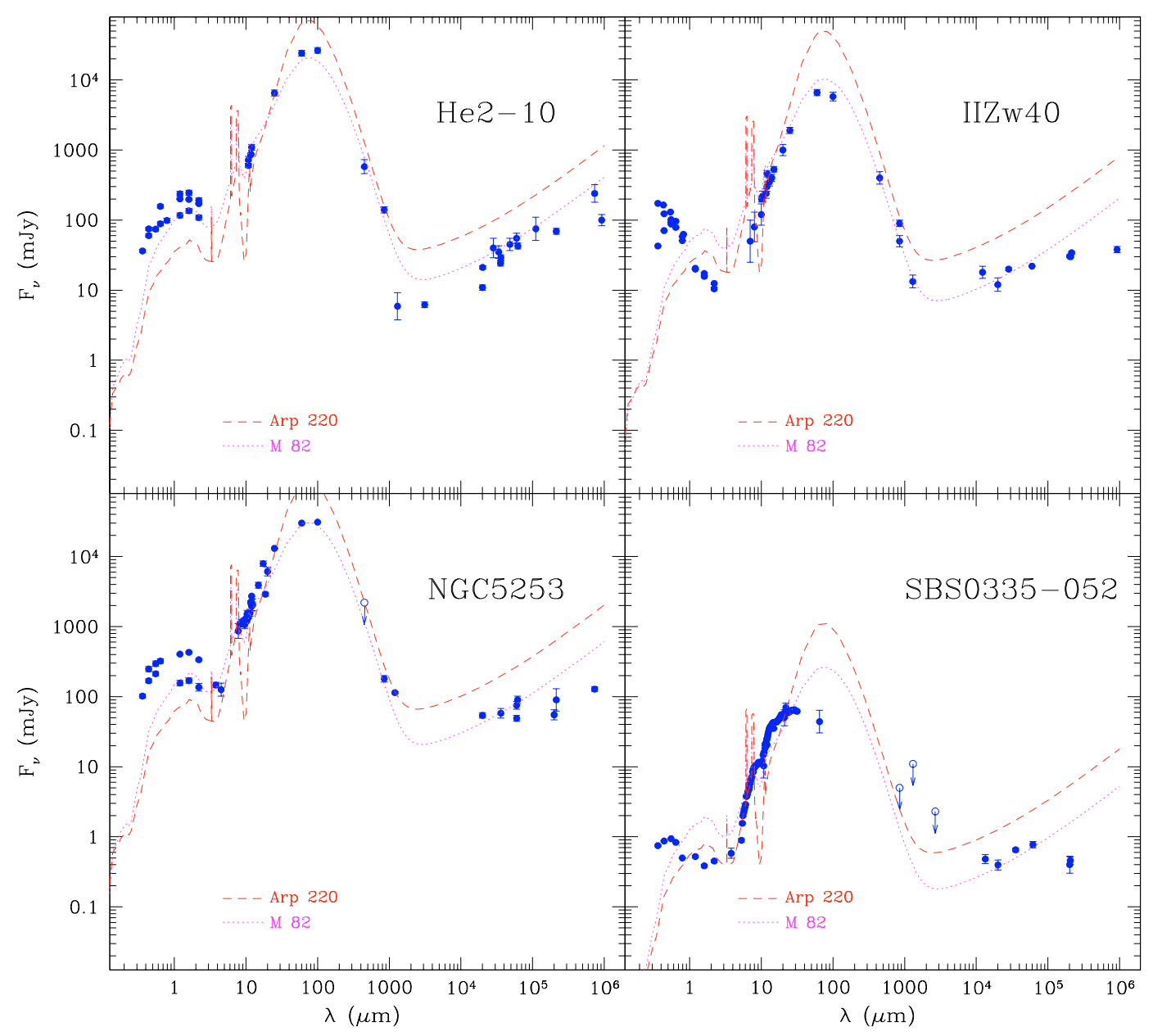

Fig. 1. Spectral flux distributions for He 2-10, II Zw 40, NGC 5253, and SBS 0335-052. Upper limits are shown as open symbols. Also plotted are the standard templates (Silva et al. 1998) for two prototypical starbursts, M 82 and Arp 220.

Table 3. Deviations from the starburst template, M 82.

\begin{tabular}{|c|c|c|c|c|c|c|c|c|c|c|c|c|c|}
\hline $\begin{array}{c}\text { Name } \\
(1)\end{array}$ & $\begin{array}{c}\text { Global RMS } \\
\text { (2) }\end{array}$ & \multicolumn{3}{|c|}{$\begin{array}{c}\text { Optical+NIR } \\
(3)\end{array}$} & \multicolumn{3}{|c|}{$\begin{array}{l}\text { MIR } \\
(4) \\
\end{array}$} & \multicolumn{3}{|c|}{$\begin{array}{c}\text { FIR-mm } \\
(5)\end{array}$} & \multicolumn{3}{|c|}{$\begin{array}{c}\text { Radio } \\
(6) \\
\end{array}$} \\
\hline I Zw 18 & $0.44(20)$ & -0.11 & 0.57 & (8) & & - & & & - & & -0.01 & 0.24 & $(10)$ \\
\hline II Zw 40 & $0.65(47)$ & 0.32 & 0.79 & $(20)$ & -0.36 & 0.48 & (13) & -0.03 & 0.16 & (6) & -0.19 & 0.28 & (8) \\
\hline SBS 0335-052 & $0.43(106)$ & -0.05 & 0.62 & (8) & 0.14 & 0.37 & (86) & -0.36 & 0.36 & (3) & -0.13 & 0.35 & (6) \\
\hline II Zw $70=$ UGC 9560 & $0.34(25)$ & 0.19 & 0.38 & $(10)$ & -0.37 & - & (1) & -0.16 & 0.19 & (2) & -0.15 & 0.24 & (11) \\
\hline NGC 5253 & $0.35(48)$ & 0.39 & 0.46 & (12) & 0.14 & 0.20 & (23) & 0.12 & 0.13 & (4) & -0.19 & 0.26 & (8) \\
\hline He $2-10$ & $0.29(41)$ & 0.20 & 0.29 & (16) & -0.05 & 0.12 & (6) & -0.09 & 0.37 & (5) & -0.17 & 0.18 & (14) \\
\hline Mrk $33=$ Haro 2 & 0.25 (29) & 0.13 & 0.24 & (13) & -0.19 & 0.08 & (2) & 0.02 & 0.15 & (3) & -0.11 & 0.24 & (10) \\
\hline
\end{tabular}

significantly in its optical+NIR emission, but the mid- and far-infrared coverage is too sparse to make an accurate comparison; this situation should be remedied by Spitzer. The remaining galaxies, Mrk 33 and II Zw 70, follow the template reasonably well.

The deviant galaxies, IIZw 40, NGC 5253, and SBS 0335-052, share several characteristics, namely: (i) they host Super Star Clusters (SSCs) (Thuan et al. 1997; Calzetti et al. 1997; Beck et al. 2002); (ii) unlike most galaxies, their FIR emission peaks at or shortward of $60 \mu \mathrm{m}$ (Vader et al. 1993; Plante \& Sauvage 2002); (iii) there are no PAH features in their MIR spectra (Roche et al. 1991; Thuan et al. 1999b; Houck et al. 2004); (iv) they have $K-L \gtrsim 1$ (Moorwood \& Glass 1982; Joy \& Lester 1988; Hunt et al. 2001); and (v) their radio spectra are rising on small spatial scales (Turner et al. 2000; Beck et al. 2002; Hunt et al. 2004). The first four characteristics are associated with what has been called an "active" mode of star formation (e.g., Hunt et al. 2002; Hirashita \& Hunt 2004). BCDs which form stars in this way have star-forming regions which are small $(\$ 100 \mathrm{pc})$ and dense $\left(n_{\mathrm{e}} \gtrsim 500 \mathrm{~cm}^{-3}\right)$. Star formation which occurs in larger, more diffuse regions was dubbed "passive". Such a 


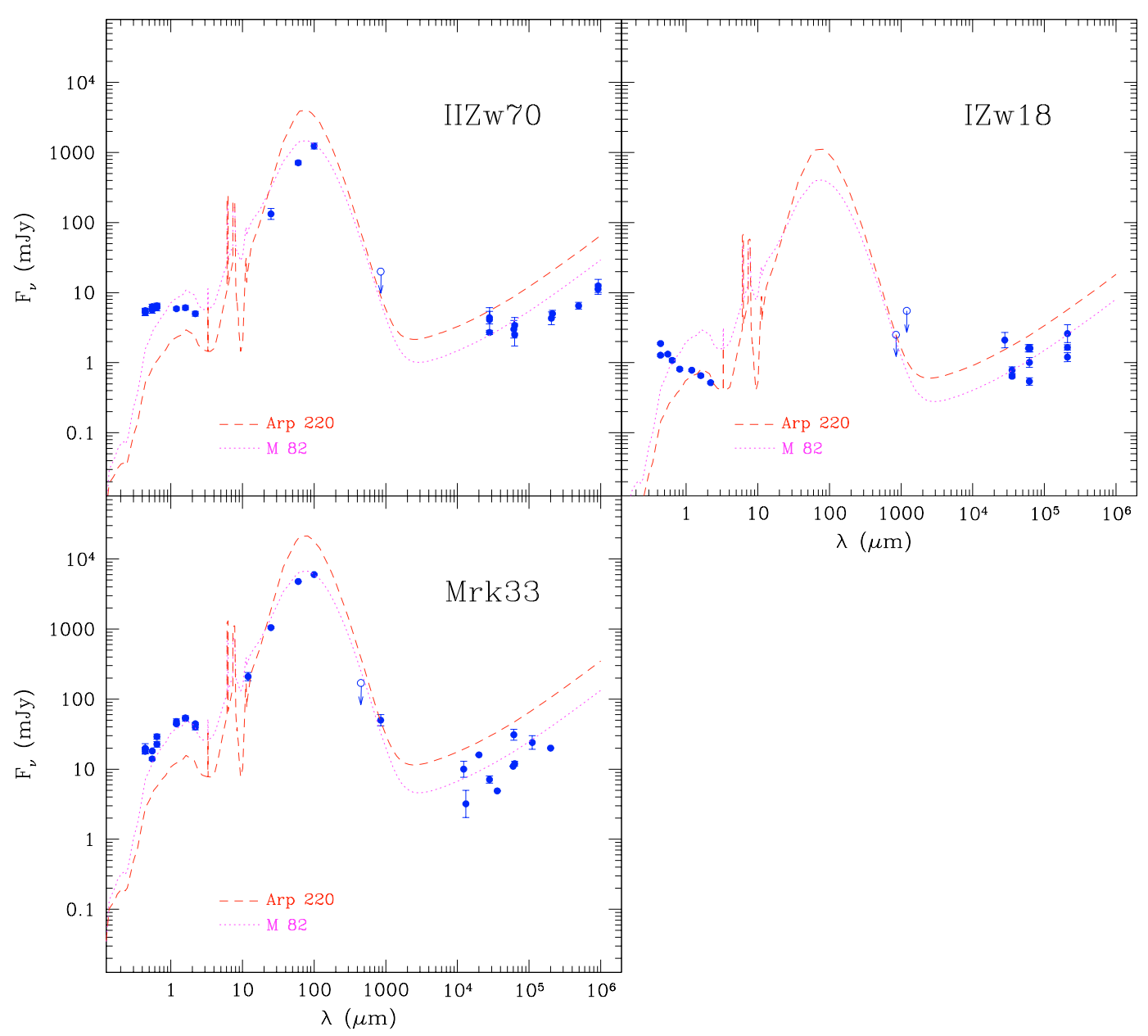

Fig. 2. Same as in Fig. 1, but for II Zw 70, IZw 18, and Mrk 33.

"dichotomy" does not occur only in low-mass low-luminosity BCDs; there is evidence that star formation is "bimodal" even in giant infrared-luminous galaxies, with more intense starbursts tending to be more heavily obscured and compact (Takagi et al. 2003).

"Active" regions tend to be more efficient at shielding ultraviolet (UV) radiation because of the greater concentration of dust. Hence the optical depth of the UV photons becomes large, and dust reaches higher temperatures than it would in more diffuse regions (Hirashita et al. 2002a). Because of the high stellar concentration and low metallicities in such BCDs, the UV radiation field is also very intense (e.g., Verma et al. 2003), which tends to suppress PAH emission (Puget \& Leger 1989). "Passive" star-forming episodes do not engender SSCs, although they can generate massive stellar clusters; dust temperatures are lower, and the MIR spectrum shows conspicuous PAH features. The deviant galaxies, II Zw 40, NGC 5253, SBS 0335-052, can all be classified as "active" BCDs; they are also characterized by compact dense star-forming complexes, as judged from HST images and optical spectra (Hunt \& Hirashita 2004, in preparation).

It is likely that the fifth trait shared by the deviant galaxies - compact radio sources with rising spectra - is also associated with active star formation. The theoretical basis for the active/passive "dichotomy" lies in the size and density of the star-forming complex (Hirashita \& Hunt 2004): compact dense regions form stars "actively" and larger more diffuse ones "passively". Rising (inverted) radio spectra surrounding individual stars, indicative of dense optically thick HII regions, are abundant in the Galaxy (Wood \& Churchwell 1989), but in external galaxies have been observed in association only with compact ( $\lesssim 10$ pc) massive star clusters (Kobulnicky \& Johnson 1999; Beck et al. 2000; Turner et al. 2000; Beck et al. 2002). The inferred densities in these small regions are extremely high, typically $\gtrsim 2000 \mathrm{~cm}^{-3}$. Hence, the two properties required for a star-forming complex to be classified as "active" are present in these inverted spectrum sources, and they may be an integral part of the phenomenon.

In addition to the three obvious "deviants", there may be another active BCD in our sample: He 2-10. It contains both SSCs (Johnson et al. 2000), and rising spectrum radio sources (Kobulnicky \& Johnson 1999). However, its FIR dust temperature is similar to normal spirals, and its $K-L$ color is $\sim 0.8$ (Glass \& Moorwood 1985), smaller than the typical values of "active" starbursts (Hunt et al. 2002). Its MIR spectrum also contains PAH features, together with a deep $10 \mu \mathrm{m}$ absorption trough (Roche et al. 1991). Hence, He 2-10 may be active on small spatial scales, but globally "passive". At solar metallicity, this galaxy is the most metal-rich in our sample. Its relatively high metal abundance may imply a more evolved ISM which 


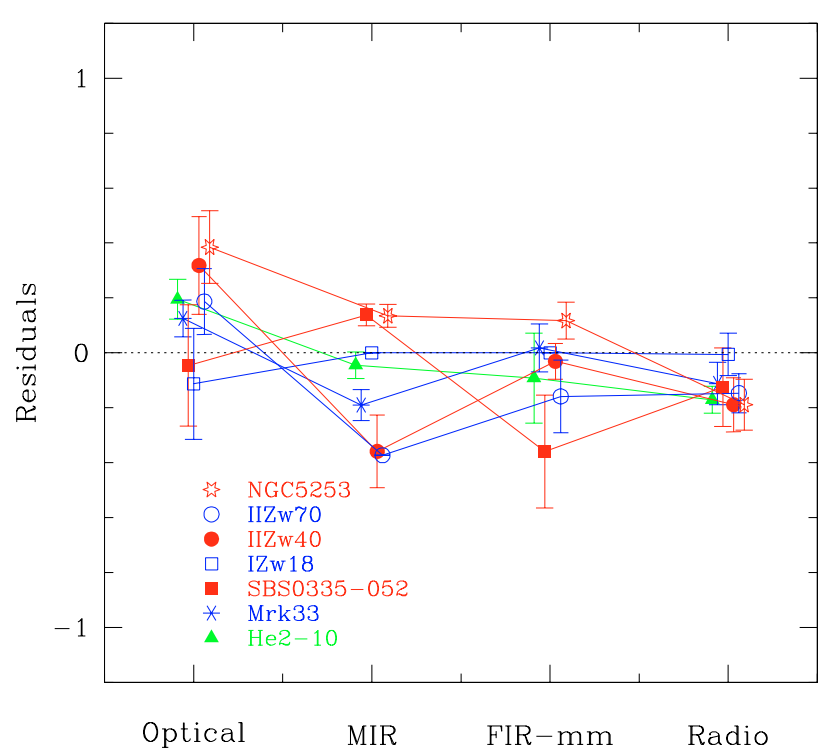

Fig. 3. The mean logarithmic residuals of each galaxy relative to the best-fit starburst template, M 82 as a function of spectral region. The exact horizontal position is arbitrary, as they have been slightly offset for better visibility. Error bars are the error in the mean, so divided by $\sqrt{n}$ relative to the values in Table 3 .

would tend to dilute "active" features and lower the dust temperatures observed over large regions.

It is as yet unclear why some regions form stars actively and others passively. Metallicity appears to have little or no influence since IZw 18 forms stars passively (Hirashita \& Hunt 2004), while SBS 0335-052 forms them actively, despite a similar (low) metal abundance. Hirashita \& Hunt (2004) speculate that high pressure and shock compression may be affect the bifurcation into active and passive regimes. Star-formation rate may also be important, since active regions tend to have higher SFRs than passive ones; however this could be an effect rather than a cause. Active regions are also invariably young ( $\$ 10 \mathrm{Myr}$ ): indeed optically thick HII regions as signified by warm dust emission and rising radio spectra appear to be the first stage of intense star formation (e.g., Beck et al. 1996; Kobulnicky \& Johnson 1999; Vacca et al. 2002). For a region to be defined as "active", it may be necessary to observe it in its early stages. Passive star formation on the other hand can be either young or more evolved, and both regimes may be associated with superbubbles or expanding shells. Indeed, with the exception of II Zw 70 which is a probable polar-ring galaxy (Cox et al. 2001), all of the galaxies in our sample contain such structures (Martin 1996; Marlowe et al. 1997; Martin 1997; Mendez \& Esteban 1997; Summers et al. 2001). Also with the exception of II Zw 70, the sample galaxies host Wolf-Rayet stars (Conti 1991; Guseva et al. 2000), implying that the starformation episodes must be younger than a few Myr (Schaerer \& Vacca 1998).

\subsection{Optical/NIR colors}

The hot dust that is associated with active star formation is revealed in the $K-L$ color, and is usually already evident in $H-K$
(Hunt et al. 2002) ${ }^{6}$. However, optical and NIR colors by themselves may be an ambiguous diagnostic of the active/passive distinction. This point can be clarified by examining the colors of our sample galaxies.

The BCDs which we have classified as "active" (NGC 5253, II Zw 40, SBS 0335-052, and possibly He 2-10) are characterized by a wide range of optical/NIR colors. The "passive" galaxies on the other hand (Mrk 33, II Zw 70, IZw 18) have uniformly blue to extremely blue optical/NIR colors. This is shown in Fig. 4 where optical/NIR colors are plotted for the sample galaxies. Also shown in the figure are the Starburst99 models for an instantaneous burst (Leitherer et al. 1999, SB99), with $1 / 20 Z_{\odot}$ to $Z_{\odot}$; the SB99 predictions include nebular continuum emission. The grid gives the observed range for spiral galaxies (de Jong 1996). The $Z_{\odot}$ curve is the one which touches the spiral galaxy grid at roughly $10 \mathrm{Myr}$ because of the onset of the asymptotic giant branch. Figure 4 shows that IZw 18, SBS 0335-052, II Zw 70, and Mrk 33 are significantly bluer than normal spirals. Only the solar metallicity SB99 models are roughly consistent with the colors (although none of them predicts the colors of IZw 18, see Hunt et al. 2003), and these only for the ages of the models, 1 Gyr or less. The remaining galaxies, NGC 5253, II Zw 40, and He 2-10, are much redder; they are only slightly bluer (NGC 5253) or consistent with the colors of early spirals. Extinction, the effect of which is shown as an arrow in the Fig. 4, would bring the colors blueward, similar to those of the "passive" BCDs. Therefore, passive BCDs seem to be associated with significantly blue colors from 0.4 to $2 \mu \mathrm{m}$, but the colors of active BCDs can vary substantially. Part of this variation may be due to dust reddening; part of it may be an age effect. With optical/NIR colors alone, this ambiguity is difficult to resolve.

The bluish or normal colors of the active BCDs are in apparent contradiction with the substantial emission at $\lambda>$ $2 \mu \mathrm{m}$ on a global scale; this is particularly striking in the case of SBS 0335-052, the prototype of an active BCD. The seeming paradox can be understood through optical depth effects and the morphology at different wavelengths. The dust extinction is so high on small scales in SBS 0335-052 ( $A_{\mathrm{V}} \gtrsim 12 \mathrm{mag}$, Thuan et al. 1999b; Plante \& Sauvage 2002) that more than half the star formation is hidden at $2 \mu \mathrm{m}$ (Hunt et al. 2001). Thus, in the optical, we are seeing only the outer shell of the star-forming regions, the inner parts of which are hidden by dust. How much of the region is visible depends on the geometry of the dust (e.g., Plante \& Sauvage 2002), and on the configuration of the emitting regions. In the optical, extended ionized gas contributes significantly to the global emission in SBS 0335-052 (Thuan et al. 1997), while in the infrared, the emission is extremely compact, confined to the most luminous star clusters (Dale et al. 2001b).

A mixture of these effects is probably responsible for the wide range of colors in what we have called "active" BCDs. In NGC 5253, a single cluster dominates the SED beyond $3 \mu \mathrm{m}$, while the entire galaxy contributes to the optical and NIR emission (Vanzi \& Sauvage 2004). The same is essentially true for

\footnotetext{
6 However, if not accompanied by a bluish $J-H$, then red $H-K$ is probably better ascribed to extinction effects.
} 

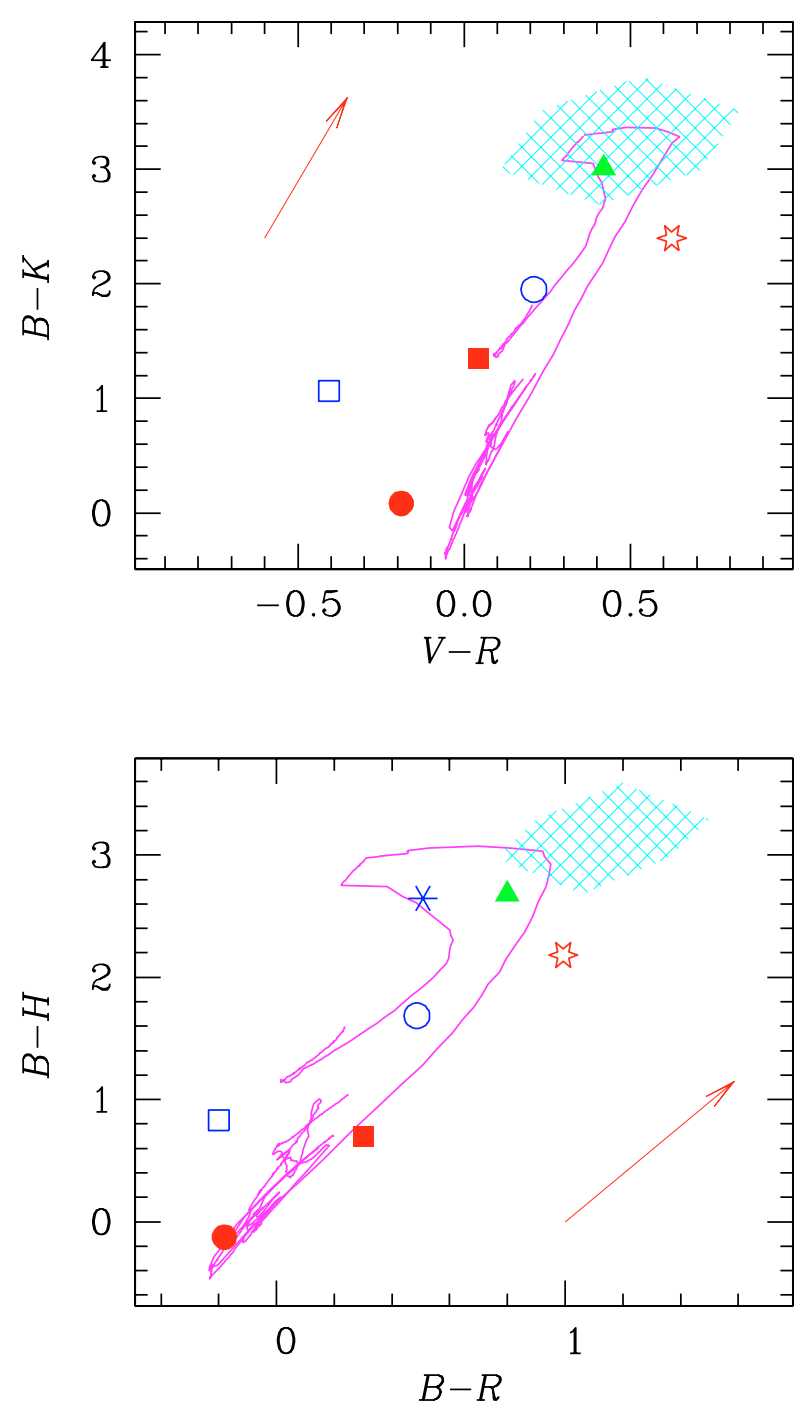

Fig. 4. Optical and optical/NIR colors of the sample galaxies. $B-K$ versus $V-R$ are shown in the upper panel, and $B-H$ vs. $B-R$ in the lower. IZw 18 is shown as an open (blue) square; II Zw 70 as an open (blue) circle; Mrk 33 as an (blue) asterix; He 2-10 as a filled (green) triangle; SBS 0335-052 as a filled (red) square; II Zw 40 as a filled (red) circle; NGC 5253 as an open (red) six-point star. The instantaneous-burst Starburst99 models (Leitherer et al. 1999) for $Z_{\odot} / 20$ to $Z_{\odot}$ are shown as (magenta) solid lines (from $1 \mathrm{Myr}$ to $1 \mathrm{Gyr}$ ), and the observed colors of spiral galaxies as a (cyan) grid (de Jong 1996). The arrow in both panels shows $A_{\mathrm{V}}=1 \mathrm{mag}$, according to the interstellar extinction curve by Cardelli et al. (1989).

II Zw 40 and $\mathrm{He} 2-10$ in which a large fraction of mid-infrared and radio emission comes from compact star clusters in a region less than 200 pc in diameter (Kobulnicky \& Johnson 1999; Beck et al. 2001; Vacca et al. 2002; Beck et al. 2002). Like SBS 0335-052, in these objects there is substantial extinction $\left(A_{\mathrm{V}} \gtrsim 10\right.$ mag, Kawara et al. 1989; Ho et al. 1990). Dust extinction is generally much lower in diffuse extended regions than in compact heavily obscured star clusters (Calzetti et al. 1997).

\section{Modelling the dust in active BCDs}

The notion that low metallicities imply low dust content in BCDs is apparently dispelled by our data. If anything, the active BCDs in our sample have a larger dust fraction relative to the radio continuum than $\mathrm{M} 82$. This is true both for warm dust at MIR wavelengths, and for cooler dust in the FIR/mm. Our new SCUBA data allow a more complete estimate of the dust mass in these BCDs, but first we need to fit the SED. To do this we have adopted DUSTY, a code which solves the radiation transport problem in a dusty environment (Ivezić \& Elitzur 1997; Ivezić et al. 1999). The solution is based on a self-consistent equation for the radiative energy density, and includes the effects of dust scattering, absorption, and emission. Such a treatment is important in the active BCDs we study here because of optical depth effects; simple screen models do not properly account for dust self-absorption. Because the code assumes thermal equilibrium, stochastic emission from very small grains (VSGs) is not considered (e.g., Desert et al. 1990). While this is not a significant problem for dust mass estimates because of the small contribution of such grains to the total dust mass, it may affect the parameters we infer from the SED fit such as temperature and dust distribution.

Because the active BCDs in our sample all host compact SSCs or ultradense HII regions or both, we have assumed a spherical shell geometry for the dust emission. The inside of the shell is exposed to radiation from a young stellar population at the appropriate metallicity and age. In general, for any geometry (Ivezić \& Elitzur 1997), the scaling properties of the radiative transfer problem leads to SED families which, for a given grain type, are governed by the spatial density distribution of the dust; the optical depth $\tau$ determines the position within the family. As first pointed out by Rowan-Robinson (1980), neither the overall luminosity nor absolute scales of density or distance are required for the exact solution; hence, the problem can be defined with relatively few quantities. With DUSTY, it is necessary to specify the temperature $T_{\text {in }}$ at the innermost boundary of the shell, and the thickness of the shell in terms of the ratio of the outer to the inner radius, $Y_{\text {out }}\left(\equiv R_{\text {out }} / R_{\text {in }}\right)$. For simplicity, we have used one-zone models, so it is necessary to specify only a single power law index $p$ for the radial run of the mass density, $R^{-p}$.

DUSTY currently only supports single-type grains, namely a single size and chemical composition. However, it allows the simulation of more realistic grain populations through the appropriate weighted means. Thus, to define a grain population, DUSTY requires the minimum and maximum grain size, $a_{\text {min }}$ and $a_{\max }$, assumed to be the same for all chemical species; the power law index $q$ describing their size distribution, $a^{-q}$; and their chemical composition. Several species are supported by DUSTY including classical silicate and graphite grains (Draine \& Lee 1984), and amorphous carbons (AMCs) (e.g., Hanner 1988; Zubko et al. 1996). The fractional number abundances must be specified for each grain type.

We implemented the SED fitting of our active BCDs by performing a grid search over various dust density distributions, $Y_{\text {out }}$, dust compositions, and $T_{\text {in }}$. This last imposes the physical size of the innermost shell boundary $R_{\text {in }}$ because of its 


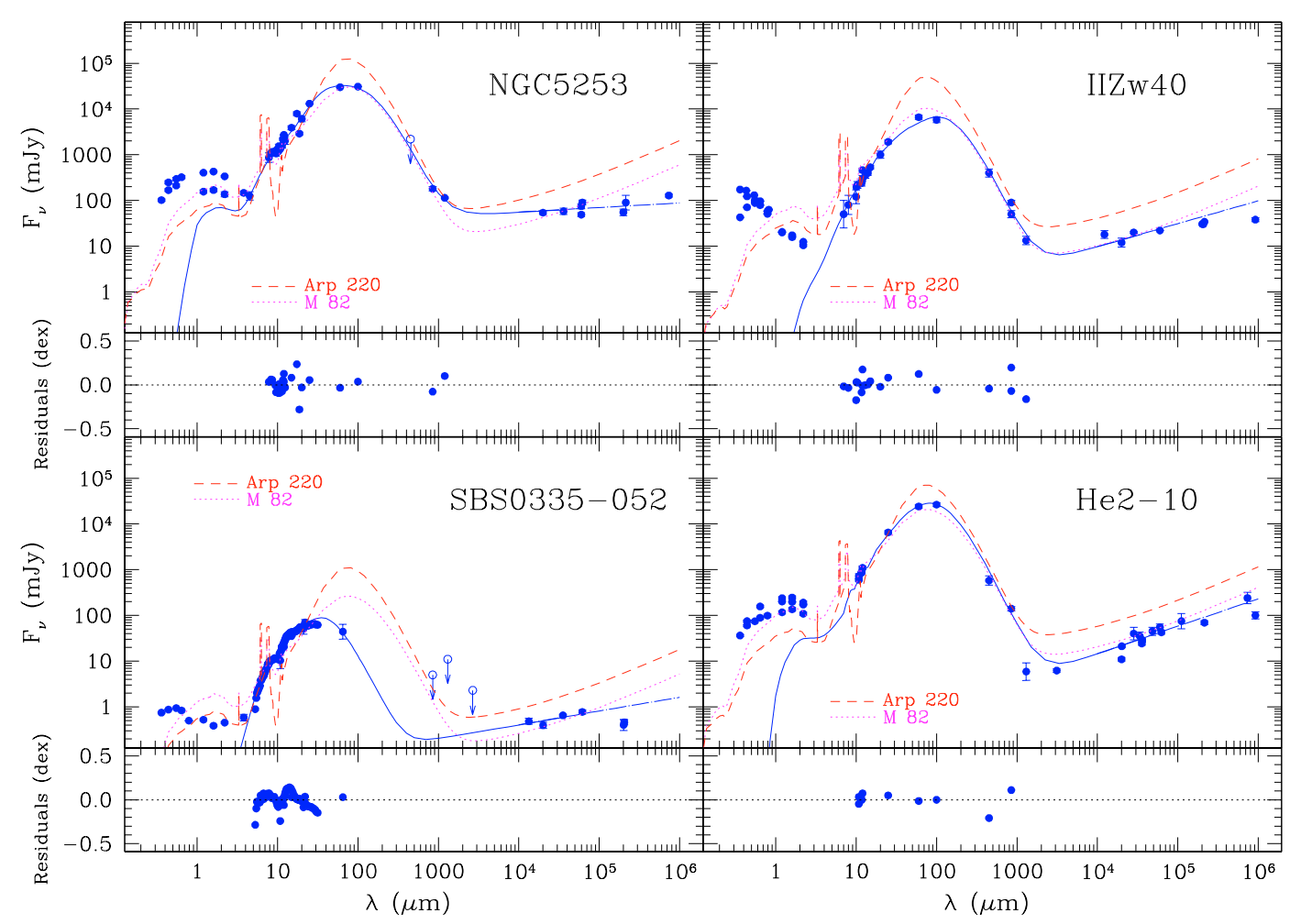

Fig. 5. Best-fitting DUSTY SED models for the "active" galaxies (upper panels shown as solid lines), together with the logarithmic residuals of the fits (lower panels). The SEDs for Arp 220 and M 82 are also shown, repeated from Figs. 1 and 2.

dependence on the intensity of the central source and the normalization of the radial coordinate. We defined three basic dust compositions for the grid search: ( $i$ ) a "standard" one (Draine \& Lee 1984); (ii) a mixture of silicates, graphite grains, and amorphous carbons (e.g., Weingartner \& Draine 2001); (iii) a silicate-free mixture with only graphite grains and amorphous carbons. Grain sizes were assumed to be distributed according to a power law, with the index $q$ fixed to -3.6 , in accordance with Weingartner \& Draine (2001), and very similar to the Mathis et al. (1977, MRN) value of -3.5 . Grain sizes were taken to be "standard" MRN, ranging from $0.005 \mu \mathrm{m}$ to $0.25 \mu \mathrm{m}$. The central exciting source spectrum was taken from SB99 for the appropriate age ( 3 Myr, see Sect. 5.1) and metallicity as determined from the optical spectra. For each metallicity $\left(1 / 20\right.$ and $\left.1 / 5 Z_{\odot}\right)$, more than 2000 DUSTY models were generated in the grid, with power-law indices $p$ ranging from 0 to $1, Y_{\text {out }}$ from 20 to 1000 logarithmically, and $T_{\text {in }}$ from 400 to $700 \mathrm{~K}$. $\tau_{V}$ was varied logarithmically from 0.1 to 100 .

Because of the importance of thermal radio emission to the observed SED, we first fit a single power law to the radio spectrum for $\lambda \geq 1 \mathrm{~cm}$. This fit was subtracted from the observed spectrum for $\lambda<1 \mathrm{~cm}$ to obtain the pure dust contribution. The best-fit DUSTY model was determined by minimizing the logarithmic residuals of the radio-subtracted dust spectrum and the DUSTY models from $5 \mu \mathrm{m}$ to $1 \mathrm{~cm}$. Once the best-fitting DUSTY model was achieved in this "coarse" grid, the parameter space of the models was refined to describe the SED as well as possible. This resulted in typically another $20-50$ models being generated. With the best-fit parameters, we also investigated the effects of varying the age of the stellar population and the grain size distribution and chemical composition.

\subsection{DUSTY model results}

The best-fitting DUSTY models are shown in Fig. 5, and the radial run of temperatures in Fig. 6. The dust parameters and radial distributions are reported in Table 4. Uncertainties in the model parameters are inferred from the grid spacing with which we searched the parameter space, together with the consequent variation in the mean logarithmic residuals. The uncertainties in the temperature at the inner shell boundary $T_{\text {in }}$ is roughly $25 \mathrm{~K}$, and that at the outer boundary $T_{\text {out }}$ on the order of a few $(2-5 \mathrm{~K})$. As for the dust mass distribution, the power-law index has an uncertainty of a few tenths, and the absolute values of the radii between 10 and $20 \%$. The dust optical depth $\tau_{V}$ is precise to $\sim 30 \%$ for the larger values $\left(\tau_{V}=30\right)$ and about $20 \%$ for the smaller ones $\left(\tau_{V} \lesssim 20\right)$.

The optical and NIR data points were not included in the fits because of unknown extinction and geometry effects. The stellar populations reflected by these data in the global SEDs are almost certainly not subject to the high extinction experienced by the central star clusters responsible for heating the dust (e.g., Calzetti et al. 1997). Indeed, Fig. 5 shows that if they were, they would not be visible at all at optical wavelengths.

Also given in Table 4 are the inferred dust masses $M_{\text {dust }}$ and infrared luminosity $L_{\mathrm{IR}}$ from $5 \mu \mathrm{m}$ to $1 \mathrm{~cm}$; this last is approximately the bolometric luminosity because of dust reprocessing of the incident UV radiation. $L_{\mathrm{IR}}$ is easily obtained by 
Table 4. DUSTY best-fit parameters for "active" BCDs.

\begin{tabular}{lccccccccccc}
\hline \hline \multicolumn{1}{c}{ Name } & $\begin{array}{c}\alpha \\
\text { (radio) }\end{array}$ & $R^{p}$ & $\begin{array}{c}T_{\text {in }} \\
(\mathrm{K})\end{array}$ & $\begin{array}{c}T_{\text {out }} \\
(\mathrm{K})\end{array}$ & $\begin{array}{c}R_{\text {in }} \\
(\mathrm{pc})\end{array}$ & $\begin{array}{c}R_{\text {out }} \\
(\mathrm{pc})\end{array}$ & $\tau_{V}$ & Dust $^{a}$ & $\begin{array}{c}L_{\mathrm{IR}} \\
\left(L_{\odot}\right)\end{array}$ & $\begin{array}{c}M_{\text {dust }} \\
\left(M_{\odot}\right)\end{array}$ & $\begin{array}{c}\langle\sigma\rangle^{b}(N) \\
(\log )\end{array}$ \\
\hline IIZw 40 & -0.5 & $R^{0}$ & 500 & 19 & 0.4 & 203 & 30 & WD+Hn & $2.5 \times 10^{9}$ & $1.3 \times 10^{5}$ & $0.10(19)$ \\
SBS 0335-052 & -0.3 & $R^{-1}$ & 400 & 69 & 0.5 & 5.4 & 30 & WD+Zb & $1.4 \times 10^{9}$ & $1.1 \times 10^{2}$ & $0.08(88)$ \\
NGC 5253 & -0.2 & $R^{-0.3}$ & 450 & 19 & 0.4 & 213 & 10 & Grf+Zb & $1.8 \times 10^{9}$ & $1.6 \times 10^{5}$ & $0.10(25)$ \\
He 2-10 & -0.6 & $R^{0}$ & 600 & 23 & 0.4 & 200 & 20 & WD & $5.6 \times 10^{9}$ & $9.6 \times 10^{4}$ & $0.09(10)$ \\
\hline
\end{tabular}

${ }^{a}$ Dust is coded by: WD+Hn (0.3 silicates, 0.4 graphite, 0.3 Hanner AMCs); WD+Zb (0.3 silicates, 0.4 graphite, 0.3 Zubko et al. AMCs); $\mathrm{Grf}+\mathrm{Zb}(0.70$ graphite, 0.3 Zubko et al. AMCs); WD ( 0.6 silicates, 0.4 graphite). All grains follow a power-law size distribution with $q=3.6$ and $a_{\min }=0.005 \mu \mathrm{m}, a_{\max }=0.25 \mu \mathrm{m}$, except for Grf $+Z \mathrm{~b}$ which has $a_{\min }=0.03$ and $a_{\max }=0.3 \mu \mathrm{m}$.

${ }^{b}$ Mean residuals from $5 \mu \mathrm{m}$ to $1 \mathrm{~cm}$.

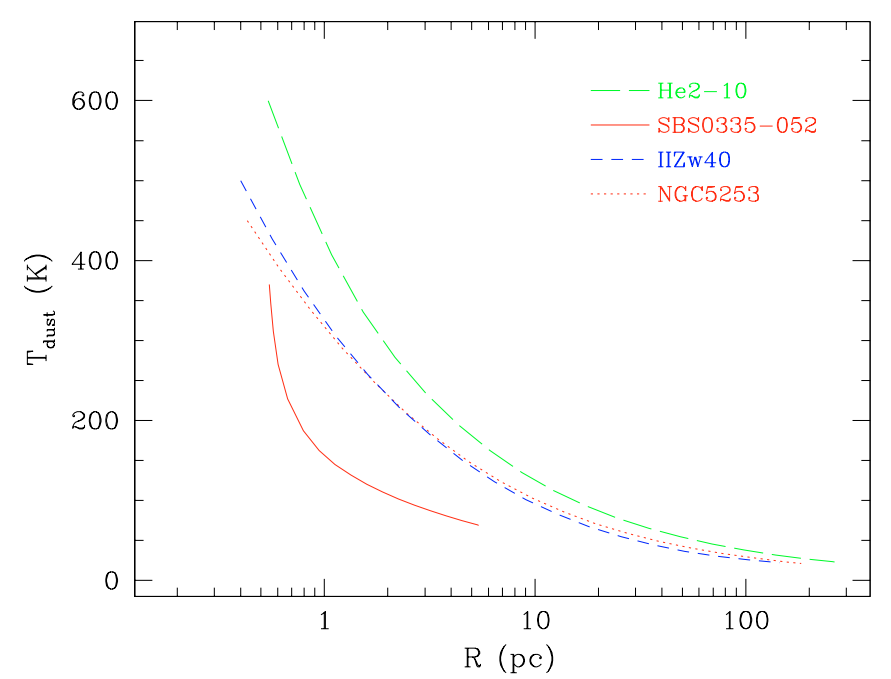

Fig. 6. Radial run of dust temperature $T_{\text {dust }}$ as predicted by the bestfit DUSTY models. SBS 0335-052 is shown by a solid (red) line, $\mathrm{He} 2-10$ by a long-dashed (green) one, IIZw 40 by a short-dashed (blue) one, and NGC 5253 by a dotted (red) one.

integrating the model over the fitted wavelength range, and applying the normalization factor used to rescale the DUSTY models to the observed SEDs. The dust mass is less straightforward to derive from the models. Although $M_{\text {dust }}$ is proportional to the optical depth $\tau_{V}$ (see e.g., Plante \& Sauvage 2002), the former depends on the amount of dust and its geometry, while the latter is also a function of dust composition, its optical properties, and the grain size distribution. Hence, converting the optical depths given by the models into dust mass involves calculating weighted averages of these quantities in the same way as in the DUSTY code. We have done this as well as possible, but the approximations for the optical quantity weighting could introduce an additional 10-20\% uncertainty in the dust masses. The equations which relate $\tau_{V}$ to $M_{\text {dust }}$ are given in Appendix B. The main uncertainty in $M_{\text {dust }}$ however is the dust geometry, that is to say the radial distribution and truncation radius $R_{\text {out }}$. From the difference between the best fit and the next best one, uncertainties in the dust mass are usually $20-30 \%$, but can be as high (in the case of SBS 0335-052) as a factor of 3 .

The most important result of the fitting procedure is the good quality of the fits: the mean (logarithmic) residuals are 0.1 or better (see Table 4). This implies that both the assumed shell geometry and the approximation of thermal equilibrium for the dust emission may not be unrealistic hypotheses. On the other hand, there could be a strong degeneracy between the temperature distribution of the dust derived from the assumption of thermal emission and the stochastic emission from VSGs. Either way the dust masses are likely to be unaffected because of the neglible contribution from the small-grain population. In II Zw 40, the fit is almost certainly affected by aperture effects since the cool-dust emission at $850 \mu \mathrm{m}$ appears extended beyond $40^{\prime \prime}$. The $230 \mathrm{GHz}$ point of $5.9 \mathrm{mJy}$ (Kobulnicky et al. 1999) for He 2-10 is impossible to reconcile with any fit and was disregarded both in the normalization and in the calculation of the residuals.

In general, with the exception of SBS 0335-052, we find that the dust mass distribution is not strongly peaked; either uniform or low-index power laws provide the best fit for the radial distribution of the dust. Again with the exception of SBS 0335-052, the star clusters modelled by our fits have rather thick shells with $R_{\text {out }} / R_{\text {in }} \sim 500$. They are also rather extended, having radii of $\sim 200 \mathrm{pc}$ (see also Vanzi \& Sauvage 2004). However, this size is deceptive, being the truncation radius of the dust shell; the dust at these radii is cool, around $20 \mathrm{~K}$ (see Table 4 and Fig. 6), and would not be detected in the MIR. The form of the SEDs at longer IR wavelengths dictates the presence of such cool dust, because of their broad peak at $\sim 60 \mu \mathrm{m}$. On the other hand, as shown by the IRS spectrum of Houck et al. (2004), the dust in SBS 0335-052 is very warm, peaking at $\sim 30 \mu \mathrm{m}$. It is also very compact; our fit gives a diameter of $\sim 10 \mathrm{pc}$, with a dust temperature of $\sim 70 \mathrm{~K}$ at this boundary. Our new $65 \mu \mathrm{m}$ ISOPHOT flux of $44 \mathrm{mJy}$ is consistent with this, but combined with the Spitzer IRS spectrum results in a much different DUSTY fit than obtained previously (Plante \& Sauvage 2002, see below).

The dust in these BCDs also appears to be relatively "standard". The same $(\sim \mathrm{MRN})$ grain size distribution and standard composition ( 0.3 silicates, 0.4 graphite grains, $0.3 \mathrm{AMCs}$ ), fits all galaxies except for NGC 5253, in which there seems to be a paucity of small grains and silicates, in agreement with Vanzi \& Sauvage (2004). AMC grains are necessary in SBS 0335-052, NGC 5253, and II Zw 40, but not in He 2-10 where a "standard" MRN composition provides the best fit. 
We found very little if any dependence of the models on either the metallicity or the age of the exciting stellar cluster. This is probably because luminosity enters into the radiative transfer problem only through the flux at the inner radius of the shell; once this is specified by the temperature $T_{\text {in }}$ and the radius $R_{\text {in }}$, the problem merely is rescaled through normalization. In all cases, we therefore adopted a $3 \mathrm{Myr}$ cluster for the final best fit, consistent with the optical spectra and the presence of Wolf-Rayet features (see Sect. 4), although we used the SB99 stellar population with the metallicity closest to that of the particular BCD.

\subsubsection{Zw 40}

Our model gives a total IR luminosity $L_{\mathrm{IR}}$ of $2.5 \times 10^{9} L_{\odot}$, in good agreement with the OB stellar luminosity $L_{\mathrm{OB}}$ of $3 \times$ $10^{9} L_{\odot}$ reported by Beck et al. (2002), and with earlier estimates (Wynn-Williams \& Becklin 1986). The structure in the starburst of II Zw 40 is however not well approximated by a single dusty shell, since high-resolution radio maps show four compact nebulae separated by 10-12 pc (Beck et al. 2002). The inner radius of $0.4 \mathrm{pc}$ given by our best-fit model is probably the best guess for the combined emission from these clusters; there is clearly warm dust (our model gives $T_{\text {in }}=500 \pm$ $50 \mathrm{~K}$ ) heated by the massive stars.

Assuming an age of 3-5 Myr, we can use the SB99 models to derive the stellar mass responsible for the IR luminosity $2.5 \times 10^{9} L_{\odot}$ gives a total stellar mass of $1.7 \times 10^{6} M_{\odot}$ associated with the starburst. This is again in good agreement with the estimate of Beck et al. (2002) who find a total of $2 \times 10^{6} M_{\odot}$ in young stars, and is $\sim 12 \%$ of the total stellar mass estimated by Vanzi et al. (1996).

The cool-dust emission is clearly extended in this galaxy, since the flux at $40^{\prime \prime}$ is about half of the global emission in the SCUBA image. Narrow-band images and long-slit spectroscopy show that the emission-line region in II Zw 40 is more extended than 40" (Vanzi et al. 1996), so the presence of spatially extended cool dust in the SCUBA image is not surprising. The diameter of $\sim 400 \mathrm{pc}$ (where $T \sim 20 \mathrm{~K}$ ) given by our model is much smaller than this $\sim 7^{\prime \prime}$; this should be however roughly the maximum extent of the dense star clusters that comprise the starburst, the core alone of which has an extent of $\sim 150 \mathrm{pc}$ (Beck et al. 2002).

The extinction given by our model of $\tau_{V}=30 \mathrm{mag}$ is significantly higher than previous estimates $\left(\tau_{V} \sim 3-5\right.$; Joy \& Lester 1988; Walsh \& Roy 1993; Vanzi et al. 1996). Part of the reason for this may be the assumption of a foreground screen by these authors. However, the discrepancy may also be ascribed to substantial optical thickness; in very dusty star clusters, even the NIR hydrogen recombination lines do not probe the central most obscured regions (e.g., SBS 0335-052; Hunt et al. 2001).

The best-fit radio index is $\alpha=-0.2$ which gives large DUSTY residuals $\langle\sigma\rangle=0.2$. We therefore steepened the radio spectral index $\alpha$ gradually to -0.5 ; the residuals monotonically decrease to 0.10 as $\alpha$ steepens. We therefore adopted $\alpha=-0.5$ which clearly does not well approximate the radio emission at lower frequencies; there is evidently substantial free-free absorption in II Zw 40 (see Beck et al. 2002).

\subsubsection{SBS $0335-052$}

Our DUSTY fit for SBS 0335-052 is substantially different than that presented by Plante \& Sauvage (2002); most importantly, it predicts a much lower dust mass and a much smaller shell. Our dust mass estimate of $1.1 \times 10^{2} M_{\odot}$ is a factor of 10 lower than by Houck et al. (2004), and $10^{3}$ times lower than previous estimates (Thuan et al. 1999b; Hunt et al. 2001; Plante \& Sauvage 2002). There is roughly a factor of 3 uncertainty on $M_{\text {dust }}$; our next best fit gives a dust mass of $\sim 3.7 \times 10^{2} M_{\odot}$ with a truncation radius $R_{\text {out }}$ of $11 \mathrm{pc}$. However, if $R_{\text {out }}$ (hence $M_{\text {dust }}$ ) are much larger, the residuals become unacceptably high. The main reason for the difference is the input SED in which we have included the Spitzer IRS spectrum given by Houck et al. (2004), and our new ISOPHOT $65 \mu \mathrm{m}$ photometry (44 mJy vs. $112 \mathrm{mJy}$ ). As pointed out by Houck et al. (2004), the spectral turnover at $\sim 30 \mu \mathrm{m}$ in the IRS data implies that the cool dust is warmer than previously thought, lowering the inferred dust mass. The IR luminosity $1.4 \times 10^{9} L_{\odot}$ of our model is also $\sim 3$ times lower than that of Plante \& Sauvage (2002), again because of the different SED. The definitive answer of how much dust is contained in the SSCs of SBS 0335-052 must wait for the long-wavelength Spitzer MIPS photometry.

The other parameters of our best-fit DUSTY model are also rather different from those by Plante \& Sauvage (2002). Although the radial fall-off of their three-zone model is the same as our one-zone $\left(R^{-1}\right)$ and the optical depths are the same $\left(\tau_{V}=30\right)$, they find a much thicker and hotter shell than we do $\left(T_{\text {in }}=700 \mathrm{~K}, Y_{\text {out }}=1000\right)$. Both of these differences can also be ascribed to the new IRS data. The smaller thickness of our model is due to the lack of cool dust emission beyond $30 \mu \mathrm{m}$; thick shells emit at longer wavelengths because of the greater abundance of cooler dust. The lower temperature of $400 \mathrm{~K}$ is dictated by the slope and relative flux of the IRS spectrum for $\lambda \lesssim 10 \mu \mathrm{m}$; hotter dust (than $\sim 500 \mathrm{~K}$ ) gives too much flux, and a slope that is not sufficiently steep. The dust parameters we find for SBS 0335-052 also differ from those found by Plante \& Sauvage (2002). Although the dust composition of both fits is similar, they find evidence for a flatter exponent in the size distribution and larger grains, while we find that it is possible to fit the SED with "standard" dust sizes.

Assuming an age of 3-5 Myr, as for II Zw 40, we can derive the total stellar mass responsible for the IR luminosity $2.8 \times 10^{9} L_{\odot}$ and find $\sim 10^{6} M_{\odot}$, only slightly lower than that found by Plante \& Sauvage (2002), $2 \times 10^{6} M_{\odot}$. It is however much lower than the $6.6 \times 10^{6} M_{\odot}$ estimate by Hunt et al. (2001) on the basis of $\operatorname{Br} \alpha$. Radio measurements have shown that the number of ionizing photons inferred from $\mathrm{Br} \alpha$ in SBS 0335-052 is inconsistently high (Hunt et al. 2004) because of the influence of stellar winds; the stellar mass given by Hunt et al. (2001) is consequently overestimated. Indeed, embedded massive star clusters and SSCs all tend to have masses around $\sim 10^{6} M_{\odot}$ (Gallagher \& Smith 1999; 
Smith \& Gallagher 2001; Vacca et al. 2002; de Grijs et al. 2004), and this value is typical of the BCDs in this paper.

\subsubsection{He 2-10}

The total $L_{\mathrm{IR}}$ of our best-fit model is $5.6 \times 10^{9} L_{\odot}$, in good agreement with the value of $6 \times 10^{9} L_{\odot}$ given by Vacca et al. (2002). Our dust mass estimate $M_{\text {dust }} \sim 10^{5} M_{\odot}$ is substantially lower than theirs, although consistent with that of Baas et al. (1994). The stellar mass inferred from SB99 assuming an age of $3 \mathrm{Myr}$ is $4 \times 10^{6} M_{\odot}$, consistent with previous estimates (e.g., Johnson et al. 2000; Vacca et al. 2002)

Vacca et al. (2002) have modelled the IR emission of He 2-10 using optically-thin shell models of the dust with a uniform density distribution. Despite the differences in the model assumptions, $R_{\text {out }}$ of our DUSTY model is similar to theirs (100-200 pc for the various emission knots). However, the inner and outer temperatures are rather different: we find $T_{\text {in }}=400 \mathrm{~K}$ and $T_{\text {out }}=23 \mathrm{~K}$, while they find a factor of two cooler dust at the inner shell boundary and warmer dust $(\sim 30 \mathrm{~K})$ at the outer boundary. They also find much lower extinction $\left(A_{\mathrm{V}} \lesssim 8\right)$ while we find $\tau_{V}=20$, a value consistent with the estimate by Phillips et al. (1984) and Kobulnicky et al. (1995). The differences in our results and those of Vacca et al. (2002) are almost certainly due to their assumption of an optically thin shell, and a single grain radius of $0.1 \mu \mathrm{m}$. The high value of $\tau_{V}$ given by our model is consistent with the observation of Vacca et al. (2002) that "none of the SSCs seen in the HST UV and optical images, or the ground-based NIR images, is detected in the Gemini $N$-band image, and vice versa".

\subsubsection{NGC 5253}

The IR luminosity of $1.8 \times 10^{9} L_{\odot}$ given by our DUSTY model is in excellent agreement with that of $1.7 \times 10^{9} L_{\odot}$ of Gorjian et al. (2001), and of Vanzi \& Sauvage (2004) after correcting for the different adopted distance. This translates into a stellar mass of $1.2 \times 10^{6} M_{\odot}$, assuming an age of 3-5 Myr and using the SB99 models as before. As mentioned previously, this age is appropriate for galaxies such as NGC 5253 with Wolf-Rayet features in their optical spectrum (Schaerer et al. 1997). Our estimate of the stellar mass agrees well with that of Calzetti et al. (1997), and again is typical of the mass of embedded clusters and SSCs (see above).

The SED of NGC 5253 has also been modelled with DUSTY by Vanzi \& Sauvage (2004). The dust mass $M_{\text {dust }}$ of $1.6 \times 10^{5} M_{\odot}$ given by our best fit is consistent with, although lower than, theirs $\left(2.3 \times 10^{5} M_{\odot}\right.$ with our Cepheid distance of $4.1 \mathrm{Mpc}$ ). The geometry of the two fits is also similar, with our one-zone best-fit model $R^{-0.3}$ compared to their two-zone distribution, $R^{0}, R^{-0.5}$. The outer shell radii also agree very well, once the different adopted distance has been accounted for ( $216 \mathrm{pc}$ vs. our value of $213 \mathrm{pc}$ ). Moreover, the two dust compositions are similar, with a lack of silicates, and $70 \%$ (us) to $80 \%$ (them) in graphite grains. The optical depth $\tau_{V}=10$ of our best-fit model is in good agreement with that of $>9 \mathrm{mag}$ measured by Calzetti et al. (1997) from optical hydrogen recombination lines, although slightly higher than $\left(A_{\mathrm{V}}=8\right)$ derived by Vanzi \& Sauvage (2004).

However, Vanzi \& Sauvage (2004) find substantially hotter dust $(570 \mathrm{~K}$ vs. $450 \mathrm{~K})$ and slightly larger grain sizes. The differences between our results and those of Vanzi \& Sauvage (2004) are almost certainly attributable to the differences in fitted grain properties. Our second-best fit (also characterized by $\langle\sigma\rangle=0.10)$ is obtained with a "standard" grain size distribution (see Sect. 5 and Table 4), and results in a dust mass $M_{\text {dust }}$ of $4.8 \times 10^{4} M_{\odot}$, with $\tau_{V}=8$ and $T_{\text {in }}=400 \mathrm{~K}$. This dust mass is more than three times smaller than that obtained with our best-fit model which has $a_{\min }=0.03 \mu \mathrm{m}$ (rather than the "standard" $a_{\min }=0.005 \mu \mathrm{m}$ ). Indeed, the mass of dust with given flux from thermalized grains depends linearly on the grain size (see e.g., Hildebrand 1983).

The uncertainties in the dust parameters used for the DUSTY fits are probably the largest contribution to the uncertainties in the derived parameters such as $T_{\text {in }}$ and $M_{\text {dust }}$. With the models and the data we have, it is difficult to determine grain size distributions. The same SED can be equally well fit by very different grain populations. UV observations and more detailed modelling of dust emission are both needed to better constrain grain size distributions and dust chemical composition.

\section{Scaling laws revisited}

As mentioned in the Introduction, there is previous evidence that neither IR nor radio luminosity accurately traces the SFR in the low-luminosity low-metallicity galaxies studied here. In this section, we examine how well the canonical correlations among MIR, far-IR, and radio luminosities are obeyed by the BCDs in our sample.

\subsection{Radio/FIR correlations}

Several previous authors have noted that the ubiquitous radio to IR correlation (e.g., Condon 1992) does not hold for some lowmetallicity dwarf galaxies (Dale et al. 2001b; Beck et al. 2002) or galaxies with young starbursts (Roussel et al. 2003). Such objects are characterized by high IR-radio ratios, and the common explanation is a lack of the synchrotron emission which typically dominates the radio continuum of luminous large spirals (e.g., Price \& Duric 1992; Roussel et al. 2003) or free-free absorption at $1.49 \mathrm{GHz}$ (Condon et al. 1991). Both of these effects are at work in the BCDs in our sample, judging from our fitted radio indices (see Table 4) and previous detailed radio observations (Kobulnicky \& Johnson 1999; Turner et al. 2000; Beck et al. 2002; Hunt et al. 2004).

First, we examine the " $q$ ratio" of the galaxies for which we have adequate data. $q$, defined by the logarithm of the ratio of the far-IR flux and the radio flux at $1.4 \mathrm{GHz}$ (Helou et al. 1985; Condon 1992), for most galaxy populations is very robust: $\langle q\rangle=2.34 \pm 0.19$ (Condon et al. 1991). He 2-10 and NGC 5253 have the highest $q$ values in our sample with $q \approx 2.6$ (see also Vacca et al. 2002; Roussel et al. 2003). These values are typical of ultraluminous infrared galaxies, thought to be powered by dense compact starbursts which are 
optically thick at $1.49 \mathrm{GHz}$ (Condon et al. 1991). The $q$ values for Mrk 33, II Zw 40, and II Zw 70 (2.5, 2.4, 2.3, respectively) are apparently representative of the normal galaxy population. SBS 0335-052 has a rather low $q, 2.0$, which is due to the reduced IR emission longward of $30 \mu \mathrm{m}$. The most "deviant" galaxies (He 2-10 and NGC 5253 on the high side, and SBS 0335-052 on the low side) differ $\gtrsim 1 \sigma$ from the mean value; thus our results neither confirm nor rule out the applicability to BCDs of the total FIR-1.4 GHz correlations. Apparently BCDs are not extreme cases globally (Roussel et al. 2003), although radio and IR emission do show strong deviations from the correlation on small spatial scales (e.g., Turner et al. 2000; Beck et al. 2002).

Next, we examine the ratios of the $60 \mu \mathrm{m}$ and radio luminosities that are necessary for the applicability of the Condon (1992) scaling relations for SFR. In order for the $60 \mu \mathrm{m}-1.4 \mathrm{GHz}$ luminosities to give the same SFR, the "standard" SED must have $f_{60 \mu \mathrm{m}} / f_{1.4 \mathrm{GHz}}=111$ (Hopkins et al. 2002). With the exception of SBS $0335-052$ and II Zw 70, all galaxies with available data exceed this value by factors of 2 to 3 . The SFRs inferred from the $60 \mu \mathrm{m}$ luminosity are thus 2 to 3 times higher than those from the radio, and several times higher (1-3 $\left.M_{\odot} \mathrm{yr}^{-1}\right)$ than the median value of $0.3 M_{\odot} \mathrm{yr}^{-1}$ found by Hopkins et al. (2002) for a similar sample of BCDs. If we assume that all the radio emission is thermal, rather than the $10 \% / 90 \%$ thermal/non-thermal mix at $1.4 \mathrm{GHz}$ (Condon 1992), we would infer even higher SFRs: $2-14 M_{\odot} \mathrm{yr}^{-1}$. Table 5 gives the SFRs inferred from the IR and radio luminosities under standard assumptions (see also Sect. 6.2). The thermal radio estimate for SFR is only valid for those BCDs such as NGC 5253, II Zw 40, and SBS 0335-052 with rather flat radio spectra. The deviations from the "standard" radio-IR correlation appear to be greater for the "active" BCDs, although our sample is small, and we have few passive galaxies with which to compare.

These results are in contrast with those of Hopkins et al. (2002) who found that SFRs inferred from $60 \mu \mathrm{m}$ and $1.4 \mathrm{GHz}$ luminosities are in close agreement for local BCDs. The main reason for the difference may be due to the paucity of data in their sample: of 50 sources, only 18 were detected with IRAS at $60 \mu \mathrm{m}$, and 23 at $1.4 \mathrm{GHz}$. Both the radio and IR data are flux-limited, with limits of 1-2 mJy for the radio surveys (NVSS and FIRST) and $200 \mathrm{mJy}$ for IRAS (Hopkins et al. 2002). The ratio of these limits is very close to the prediction of the IR-radio correlation, perhaps making it possible to mimic the common trend. Moreover, few of the lowest-metallicity galaxies are detected at both IR and radio wavelengths. Of the $\sim 20$ BCDs in their sample with $Z<Z_{\odot} / 10$, only three have both detections; two of these three have IR-inferred SFRs greater than radio ones.

The extremely metal poor "active" BCD SBS 0335-052 apparently does follow the IR-radio correlation; the SFRs inferred from the $60 \mu \mathrm{m}$ and $1.4 \mathrm{GHz}$ luminosities are the same, $0.2 M_{\odot} \mathrm{yr}^{-1}$. However, the true SFR is $\sim 1 M_{\odot} \mathrm{yr}^{-1}$ (Hunt et al. 2004). The $60 \mu \mathrm{m}$ luminosity is anomalously low because of the absence of cool dust, and the radio emission is low because of the strong thermal contribution and free-free absorption at $1.4 \mathrm{GHz}$. This is yet another aspect of the "conspiracy" described by Bell (2003): in low-luminosity dwarfs, "the radio-IR correlation is linear not because both radio and IR emissions track SFR, but rather because both radio and IR emissions fail to track SFR in independent, but coincidentally quite similar, ways" (see also Price \& Duric 1992).

Starburst age can be another factor in determining the validity of the IR-radio correlations, and the ability of these luminosities to accurately trace SFR. In their studies of starforming galaxies, Roussel et al. (2003) and Cannon \& Skillman (2004) have found that the radio continuum emission in young $(<10 \mathrm{Myr})$ starbursts tends to be pure thermal. Indeed, Cannon $\&$ Skillman (2004) have suggested that the observed radio spectral index be used as an age indicator. Heavily embedded star clusters will have positive spectral indices at ages $\lesssim 1 \mathrm{Myr}$, associated with optically thick thermal bremsstrahlung (e.g., Beck et al. 2000). Then as they become increasingly optically thin, the spectral index will flatten to -0.1 . When supernovae (SN) and supernova remnants (SNRs) begin to contribute to the radio emission after $\sim 10 \mathrm{Myr}$, the radio index will steepen. Evolved starbursts in spiral galaxies in which cosmic-ray diffusion processes have taken over ( $\gtrsim 50-100 \mathrm{Myr})$ will have the "canonical" radio spectral index of $\sim-0.8$ (Condon 1992).

\subsection{MIR/FIR correlations}

The availability of ISO data for large numbers of galaxies has prompted the use of MIR luminosities to trace star formation (e.g., Roussel et al. 2001; Förster Schreiber et al. 2004). Moreover, the relative constancy of the 7 to $15 \mu \mathrm{m}$ ratio in normal star-forming galaxies has led to the formulation of "standard" SEDs, and the potential application to high-redshift sources (Dale et al. 2001a; Dale \& Helou 2002). The good correlation between PAH strength and cold dust in normal starforming disks (Haas et al. 2002) is one of the tenets of such formulations, but it is not clear that PAH strength can be used to unambiguously trace star formation. In Sect. 3, we found that the "active" BCDs were not well fit by the starburst template, M 82. Here we examine the implications in the context of MIR-diagnostics of SFRs and MIR flux ratios.

Because of the absence of PAH features or AFEs in the active BCDs, and the presence of warm dust, such galaxies do not follow the usual trends for MIR emission. In NGC 5253, II Zw 40, and SBS 0335-052, the 15 to $7 \mu \mathrm{m}$ flux ratio is $\gtrsim 3$ times larger than would be expected from the correlations presented in Roussel et al. (2001). These authors emphasize that their MIR correlations are only applicable in star-forming regions which are dominated by AFEs, but without spectral information it is difficult to assess this a priori. Moreover, the ratio of 15 to $850 \mu \mathrm{m}$ flux in NGC 5253, II Zw 40, and He 2-10 exceeds that predicted by the Dale \& Helou (2002) models by a factor of 3; in SBS 0335-052, where we have no SCUBA detection, even the lower limit is greater than the "standard" prediction. We have no data for the $7 \mu \mathrm{m}$ flux of $\mathrm{He} 2-10$, but the remaining active BCDs show a similar trend for the 7 to $850 \mu \mathrm{m}$ flux ratio.

Again because of the suppression of AFEs in the active BCDs, the SFRs predicted by their $7 \mu$ m luminosity (Roussel et al. 2001) are factors of $\gtrsim 9$ lower than those predicted by 
Table 5. SFRs ${ }^{a}$ from various luminosity indicators.

\begin{tabular}{lccccc}
\hline \multicolumn{1}{c}{ Name } & $S F R_{7 \mu \mathrm{m}}^{b}$ & $S F R_{15 \mu \mathrm{m}}^{b}$ & $S F R_{60 \mu \mathrm{m}}^{c}$ & $S F R_{1.4 \mathrm{GHz}}^{d}$ & \begin{tabular}{c}
$S F R_{1.4 \mathrm{GHz}}^{e}$ \\
\multicolumn{1}{c}{$(1)$}
\end{tabular} \\
$(2)$ & $(3)$ & $(4)$ & $(5)$ & $(6)$ \\
\hline IZw 18 & - & - & - & 0.04 & 0.35 \\
II Zw 40 & 0.1 & 1.1 & 1.2 & 0.6 & 5.4 \\
SBS 0335-052 & 0.2 & 1.8 & 0.2 & 0.2 & 1.8 \\
II Zw 70 = UGC 9560 & - & - & 0.4 & 0.3 & 2.4 \\
NGC 5253 & 0.1 & 0.9 & 0.7 & 0.2 & 1.9 \\
Mrk 33 = Haro 2 & - & - & 3.4 & 1.6 & 13.5 \\
He 2-10 & - & 2.6 & 2.5 & 0.8 & 6.9 \\
\hline
\end{tabular}

\footnotetext{
${ }^{a} M_{\odot} \mathrm{yr}^{-1}$.

${ }^{b}$ Assuming the relations in Roussel et al. (2001).

${ }^{c}$ Total SFR, assuming the relation in Hopkins et al. (2002).

${ }^{d}$ Total SFR, assuming the relations in Condon (1992).

${ }^{e}$ Assuming the radio emission is purely thermal free-free at $10000 \mathrm{~K}$ (Condon 1992).
}

the (thermal) radio emission. In II Zw 40, the deficit is $\gtrsim 50$. Their $7 \mu \mathrm{m}$ SFRs also do not compare well with those inferred from the $15 \mu \mathrm{m}$ luminosities, and can be as much as a factor of $\gtrsim 10$ lower. Table 5 gives the SFRs inferred from the MIR, FIR, and radio luminosities, and enables a comparison of the different indicators which hold for other samples. It is clear that the active BCDs in our sample are highly discrepant, relative to normal star-forming galaxies. On the other hand, certainly II Zw 70 and probably Mrk 33 show SFRs that scale as usual.

Another assessment of the validity of "standard" SEDs to BCDs is the $24 \mu \mathrm{m} q$ ratio (Appleton et al. 2004). The $24 \mu \mathrm{m}$-to-radio correlations in the Spitzer Extragalactic First Look Survey give a mean ratio at zero redshift of $q_{24}=$ $\log \left(F_{24} / F_{1.4 \mathrm{GHz}}\right)=0.84 \pm 0.28$. For the 4 active BCDs in our sample, this ratio is exceeded at levels of $\gtrsim 4 \sigma:\left\langle q_{24}\right\rangle=2.0 \pm$ 0.16. Mrk 33 and II Zw 70 have lower $q_{24},\left\langle q_{24}\right\rangle=1.6$, still higher than the mean value. Appleton et al. (2004) find a larger dispersion in $q_{24}$ than in the equivalent $70 \mu \mathrm{m}$ value, and suggest that there is much more intrinsic dispersion in the IR/radio ratio at $24 \mu \mathrm{m}$ than at longer wavelengths. The SED variations of the kind observed in our sample, due either to active/passive dichotomy or to metallicity, may be causes of such dispersion.

\section{Age and dust masses}

In Sect. 6.1, we discussed the hypothesis of Cannon \& Skillman (2004) that the shape of the radio spectrum indicates starburst age. The IR portion of the spectrum also depends on the age of the starburst because of the time variation of dust self-absorption and the UV radiation field (e.g., Takeuchi et al. 2003). The effects of metallicity are more difficult to assess, both because of the small size of our sample and because of the dearth of data for the "passive" BCDs. Nevertheless, we can use our sample to gain insight into possible dust-formation mechanisms and evolution.

The origin of dust in galaxies is directly connected to their star-formation history (SFH); dust is ultimately produced by the various byproducts of stellar evolution. In very young
( $\$ 100 \mathrm{Myr}$ ) or primordial starbursts, type II supernovae (SNe) are the only possible dust production mechanism. Later, hot massive stars (e.g., Wolf-Rayet) and, still later, cooler less massive stars (Asymptotic Giant Branch, AGBs) condense dust in their stellar winds. Dust production in these various phases has been estimated by Morgan \& Edmunds (2003); at least $100 \mathrm{Myr}$ are needed to achieve the conditions for dust nucleation in the stellar evolution of low- and intermediate-mass stars.

We wish to examine whether the dust masses inferred from our DUSTY SED models can be attributed to the current starburst, and whether metallicity plays a role. To do so, we neglect the contribution of AGBs, which at $100 \mathrm{Myr}$ are roughly $1 \%$ of the total dust yield (Morgan \& Edmunds 2003). We also neglect Wolf-Rayet stars (Morgan \& Edmunds 2003), because such massive stars are rare, and their dust yield is low, $\sim 10^{-5} M_{\odot} /$ star (Marchenko et al. 2002). We therefore use the models of Hirashita et al. (2002a), based on the dust yields from type II SNe given by Todini \& Ferrara (2001), to estimate the amount of dust generated by the current burst. However, we first need an estimate of SFRs. Because of the difficulties described in Sect. 6, it is not clear how we can reliably deduce SFRs for the galaxies in our sample. As a "best guess", we adopt the thermal radio estimate from Col. 6 in Table 5 for the three galaxies with approximately flat radio spectral indices (II Zw 40, SBS 0335-052, NGC 5253), and the $60 \mu \mathrm{m}$ estimate (Col. 4) for the remaining ones.

At ages of 3-5 Myr, with the exception of SBS 0335-052, type II SNe are not capable of producing the amount of dust inferred from our models; at $5 \mathrm{Myr}$, we would expect $\gtrsim 2 \times$ $10^{3} M_{\odot}$ of dust from the Hirashita et al. (2002a) models and $\gtrsim 10^{2} M_{\odot}$ from those by Morgan \& Edmunds (2003). These masses are 100-1000 times lower than the $10^{5} M_{\odot}$ we find from our models of the active BCDs. Because of the presence of Wolf-Rayet stars (see Sect. 4), the present burst in all galaxies except II Zw 70 must be young; but with the exception of SBS 0335-052, all the galaxies for which we have dust mass estimates have NIR colors which indicate an evolved stellar population. Hence, the dust heated by the present burst almost 
certainly results from previous episodes of star formation. For the SFRs of our sample, the dust yields from $\mathrm{SNe}$ alone at or beyond ages of $\$ 30 \mathrm{Myr}$ are more than sufficient to produce the $\sim 10^{5} M_{\odot}$ of dust inferred from our models (Hirashita et al. 2002a; Morgan \& Edmunds 2003).

On the other hand, the low dust mass we infer for SBS 0335-052 can easily be ascribed to the current young burst. Indeed, the dust yields are rather high for the $\sim 10^{2} M_{\odot}$ of dust inferred from our SED. In this BCD the uncertainty in the SED itself makes the dust estimate particularly problematic. However, should our dust mass be correct, the burst could be extremely young ( $\$ 2 \mathrm{Myr}$ ), even though the radio spectral index is slightly nonthermal ( $\sim 0.3)$. Alternatively, either the dust production of a single type II SN could be lower than that assumed by Hirashita et al. (2002a), or other mechanisms could be important such as outflows (Lisenfeld \& Ferrara 1998) or dust destruction by SNe shocks (e.g., Dwek \& Scalo 1980). Obviously on the basis of one galaxy it is impossible to ascertain whether metallicity, youth, or compact size/density (the "active/passive" dichotomy) are the primary factors in shaping the unusual SED of SBS 0335-052. More MIR and FIR observations of low-metallicity BCDs are needed to resolve the question.

\section{Metallicity and dust-to-gas ratio}

One of the foundations of models of dust formation and evolution in galaxies has been the dust-to-gas ratio $\mathcal{D}$ (Lisenfeld \& Ferrara 1998; Edmunds 2001; James et al. 2002; Hirashita et al. 2002b). Here we evaluate $\mathcal{D}$ for our sample galaxies with the aim of reassessing trends of $\mathcal{D}$ with metallicity.

The gas mass in low-metallicity BCDs consists primarily of atomic hydrogen. Molecular gas in the form of $\mathrm{CO}$ is notoriously difficult to detect in low-metallicity objects, and of our sample only the highest metallicity objects, NGC 5253, He 2-10, Mrk 33, have been detected in the $\mathrm{CO}(1-0)$ transition (Baas et al. 1994; Kobulnicky et al. 1995; Barone et al. 2000; Meier et al. 2001, 2002; Bravo-Alfaro et al. 2004). NGC 5253 has also been detected in a MIR $\mathrm{H}_{2}$ line with ISO, although II Zw 40 was not (Rigopoulou et al. 2002). These CO measurements together with HI masses taken from the literature (Kobulnicky \& Skillman 1995; van Zee et al. 1998; Thuan et al. 1999a; Kobulnicky \& Skillman 1996) enable us to calculate total gas masses. The dust-to-gas ratio $\mathcal{D}$ is then derived by combining our model results for dust mass, and reporting all quantities to our assumed distance. The molecular gas mass in NGC 5253 is $0.4 \%$ that of the atomic gas (Meier et al. 2002), but more than twice the atomic gas mass in the solar-metallicity galaxy He 2-10 (Kobulnicky et al. 1995). The lack of CO detections in IIZw 40 and SBS 0335-052 could imply that the gas in these BCDs is entirely atomic, or that $\mathrm{CO}$ is not a good tracer of $\mathrm{H}_{2}$ at these sub-solar metallicities (e.g., Taylor et al. 1998).

Figure 7 shows graphically the known correlation between $\mathcal{D}$ and oxygen abundance (Issa et al. 1990); our new determinations are shown as open stars, data from the literature as

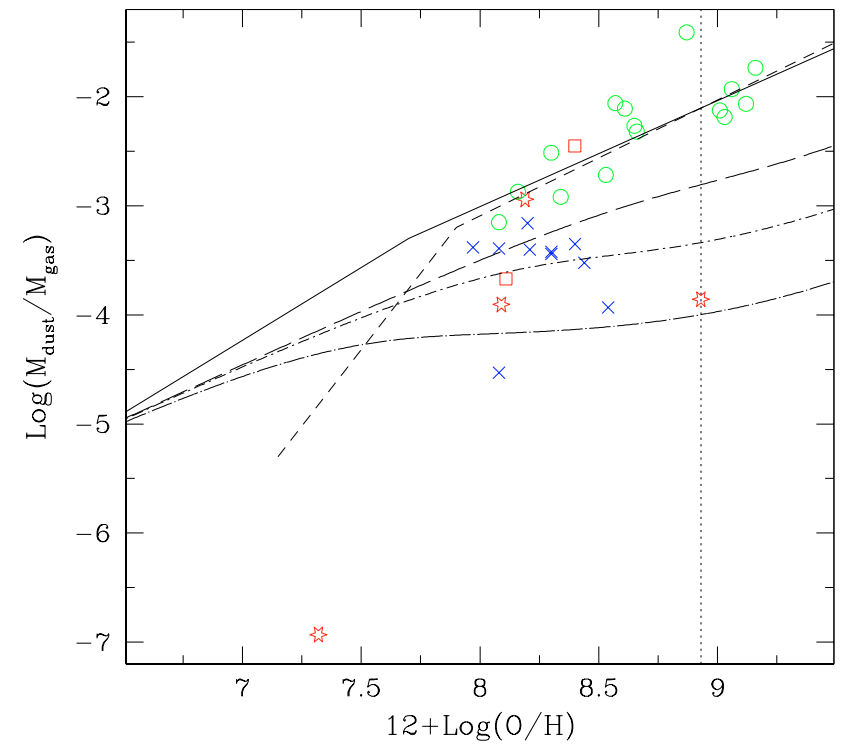

Fig. 7. Dust-to-gas mass ratios $\log \mathcal{D}$ versus oxygen abundance $12+$ $\log (\mathrm{O} / \mathrm{H})$. The four galaxies for which we have DUSTY models and thus dust masses are shown as open stars; other data from the literature are also plotted (open circles are from James et al. 2002; and $\times$ from Lisenfeld \& Ferrara 1998). Open squares show data for II Zw 70 (Lisenfeld \& Ferrara 1998) and Mrk 33 (James et al. 2002), both of which are in our sample. The vertical dotted line indicates solar metallicity. The solid and short-dashed lines are the models by James et al. (2002), and the long-dashed and dot-dashed lines the models for various dust destruction efficiencies by Hirashita et al. (2002b).

open circles (James et al. 2002) and $\times$ (Lisenfeld \& Ferrara $1998)^{7}$; Seyfert galaxies and galaxies for which there are no IRAS detections are not included. We have also indicated with open squares the two galaxies in our sample which follow the standard starburst template, and for which we did not have sufficient data to perform a DUSTY fit: II Zw 70 (Lisenfeld \& Ferrara 1998) and Mrk 33 (James et al. 2002). NGC 5253 appears in the James et al. (2002) sample with $\log \mathcal{D}=-2.92$, in very good agreement with our value $\log \mathcal{D}=-2.94$.

Model predictions for the $\mathcal{D}$-metallicity correlation are also shown in Fig. 7 (Edmunds 2001; Hirashita et al. 2002b). The Hirashita et al. (2002b) models, shown as long-dashed and dot-dashed curves, vary because of the dust destruction efficiency which begins to be effective when oxygen abundance $12+\log (\mathrm{O} / \mathrm{H})$ reaches $\sim 8$. Their models neglect outflow, and are complementary to Lisenfeld \& Ferrara (1998) who instead attribute the variation in $\mathcal{D}$ to variations in the mass outflow rate. Both the Lisenfeld \& Ferrara (1998) and Hirashita et al. (2002b) models predict a non-linear trend for $\mathcal{D}-\mathrm{O} / \mathrm{H}$, unlike Edmunds (2001) and James et al. (2002) who predict a linear one, proposing that the interstellar dust mass is an approximately constant fraction of the ISM metal abundance. A solid line indicates their prediction for dust production by $\mathrm{SNe}$, and the short-dashed one represents that by only evolved lowand intermediate-mass stars. It is apparent from Fig. 7 that none of the models reproduces the trends of $\mathcal{D}-\mathrm{O} / \mathrm{H}$ observed in our and other BCD samples. James et al. (2002) argue that

${ }^{7}$ Following Hirashita et al. (2002b), we have eliminated their factor of 0.5 in their $\mathrm{HI}$ mass derivation. 
Lisenfeld \& Ferrara (1998) underestimated the cool-dust component because of the unavailability of sub-mm fluxes; this would push the $\times$ 's upwards in Fig. 7. Nevertheless, our estimates of $\mathcal{D}$ which take into account the cool dust in a selfconsistent way are equally deviant from a linear model. In addition to outflow and dust destruction mechanisms, intermittent SFH and age probably play important roles in governing $\mathcal{D}$ (Hirashita et al. 2002b). However, without significantly more data at low metallicities $(12+\log (\mathrm{O} / \mathrm{H}) \lesssim 8)$, it will be difficult to decide between the linear/non-linear alternatives. As pointed out by James et al. (2002), dust detections in low-metallicity star forming galaxies are badly needed.

\section{Implications for photometric redshifts}

The determination of the spectroscopic redshift of distant submm/mm sources has been extremely challenging due to their very weak and uncertain optical counterparts. However, the problem of the redshift determination has been partially solved by using their far-IR - to - radio SED, compared to local templates, to obtain a photometric redshift (Yun \& Carilli 2002; Hughes et al. 2002). This method has been tested to successfully recover the spectroscopic redshifts with a reasonable accuracy $\left(z_{\text {phot }}-z_{\text {spec }}<0.3\right.$, at $\left.1<z<3\right)$. Generally, even the simple submm-to-radio spectral index $\left(\alpha_{1.4 \mathrm{GHz}}^{350 \mathrm{GHz}}\right)$ provides a good approximation of the actual redshift (Carilli \& Yun 1999). These radio-submm photometric redshifts have been calibrated by using templates of local, massive and chemically evolved starburst galaxies (e.g. M 82, Arp 220; here we focus on the non-AGN cases), which are characterized by far-IR bumps peaking at $\sim 60-100 \mu \mathrm{m}$ and a radio tail dominated by synchrotron emission. Such templates are certainly adequate for the submm-mm sources at $z \sim 2.5$ identified by SCUBA and MAMBO (Chapman et al. 2003; Dannerbauer et al. 2004), which are massive and metal-rich (e.g., Tecza et al. 2004; Genzel et al. 2003). However, the starburst galaxies which will be detected at very high redshift $(z \geq 6)$ by the forthcoming submm facilities (and ALMA in particular) will probably be characterized by compact star forming regions and low metallicities, similar to some of the local BCDs discussed in this paper. As consequence, the SED of primordial galaxies at $z>6$ will likely be characterized by warmer infrared bumps and thermal radio emission. The use of "standard" (M 82-like) SEDs applied to primordial galaxies with SEDs similar to the BCDs in our sample would yield deceptively high, significantly overestimated photometric redshifts (up to a factor of 2).

Another related factor which is likely to affect the SED of starburst galaxies at $z>6$ is that at such early epochs (age of the universe $<1$ Gyr) AGB stars have not yet evolved to produce dust. At $z>6$ the only viable mechanism for dust production is through type II supernovae (Maiolino et al. 2004). SN dust has different properties than "standard" dust (smaller grains), resulting in warmer SEDs. As discussed in Sect. 8, a SN component of dust is also likely to contribute to the warm SED of the BCD SBS 0335-052 (Hirashita et al. 2002a). Photometric redshifts for primordial galaxies calibrated by using the SEDs of local BCDs will be presented in a forthcoming paper.

\section{Conclusions}

We have used new and archival data to derive the optical-to-radio spectral energy distributions for a sample of seven low-metallicity BCDs. Such galaxies are regarded as the best local laboratories for high redshift, primordial galaxies, forming stars for the first time. In particular, their infrared and radio emission provide important constraints on the early stages of star formation and on the associated dust properties.

The SEDs of BCDs have revealed a wealth of information as well as unexpected, important properties. The main results can be summarized as follows:

1. The BCD SEDs deviate significantly from the standard templates of "classical", evolved and massive starburst galaxies in several ways. In particular: (i) the radio spectrum is generally flatter, indicative of thermal emission; (ii) the shape of the infrared bump generally implies warmer dust temperatures (with SBS 0335-052 being an extreme case in this respect); (iii) the mid-IR spectra show little or no Aromatic Features in Emission (e.g., PAHs).

2. The different spectral properties of BCDs relative to "normal" starburst galaxies also yield strong deviations from the "scaling laws". In particular, BCDs deviate substantially from the radio/FIR and MIR/FIR relations. One of the consequences of such deviations is that the SFR inferred from any of these indicators (radio, FIR, MIR) may be wrong by large factors.

3. The origin of the different SEDs of BCDs with respect to "classical" starburst galaxies is not clear yet. Very likely the very compact and dense nature of the star forming regions in some BCDs (the "active" ones) plays a major role, and is probably responsible for heating the dust to higher temperatures and for the absence of AFEs. Age may also be important, particularly in the dominance of the thermal emission over synchrotron in the radio. A young age is also probably related to the compactness of the star forming regions discussed above. The different origin of dust in the early stages of star formation (supernovae vs. evolved stars), resulting in different dust properties (typically smaller grains), is another factor which is likely to play an important role in the shape of the IR SEDs. The influence of metallicity is not clear, and additional data are definitely required to further investigate its effects on the SEDs of star-forming galaxies.

4. We have modelled the IR SEDs by means of a dust radiative transfer code, which has allowed us to derive the infrared luminosities, dust radial distribution, masses and temperatures. An important result is that, with the notable exception of SBS 0335-052, the observed dust mass cannot be accounted for by the dust produced during the short episode of star formation traced by the still ongoing burst; most of the dust must have been produced by supernovae or AGB stars from previous episodes of star formation. Another important result is that the dust-to-gas ratio is not a linear function of the metallicity, nor does the observed relation follow the prediction of any of the more sophisticated models. The latter result suggests that the dust production mechanisms in different metallicity environments need to be reconsidered. 
5. Finally, the significant deviations of the BCD SEDs with respect to "classical" evolved and massive starburst galaxies prompt for a revision of the photometric redshift techniques to be applied to the primordial, very high redshift galaxies, expected to be detected by facilities such as ALMA and SKA.

Acknowledgements. We warmly thank F. Bertoldi and K. Menten who made available their MAMBO measurement of $\mathrm{IZw} 18$. We are also grateful to an astute referee whose comments helped improve the paper, and to F. Galliano who brought an important measurement error to our attention during the proof verification. This research has made use of the NASA/IPAC Extragalactic Database (NED) which is operated by the Jet Propulsion Laboratory, California Institute of Technology, under contract with the National Aeronautics and Space Administration. We also acknowledge the Canadian Astronomy Data Centre, which is operated by the Dominion Astrophysical Observatory for the National Research Council of Canada's Herzberg Institute of Astrophysics.

\section{References}

Anders, E., \& Grevesse, N. 1989, Geochimica \& Cosmochimica Acta, 53, 197

Appleton, P. N., Fadda, D. T., Marleau, F. R., et al. 2004, ApJS, 154, 147

Baas, F., Israel, F. P., \& Koornneef, J. 1994, A\&A, 284, 403

Barone, L. T., Heithausen, A., Hüttemeister, S., Fritz, T., \& Klein, U. 2000, MNRAS, 317, 649

Baugh, C. M., Cole, S., Frenk, C. S., \& Lacey, C. G. 1998, ApJ, 498, 504

Beck, S. C., Turner, J. L., Ho, P. T. P., Lacy, J. H., \& Kelly, D. M. 1996, ApJ, 457, 610

Beck, S. C., Turner, J. L., \& Kovo, O. 2000, AJ, 120, 244

Beck, S. C., Turner, J. L., \& Gorjian, V. 2001, AJ, 122, 1365

Beck, S. C., Turner, J. L., Langland-Shula, L. E., et al. 2002, AJ, 124, 2516

Beckwith, S., Evans, N. J., Becklin, E. E., \& Neugebauer, G. 1976, ApJ, 208, 390

Bell, E. F. 2003, ApJ, 586, 794

Bessell, M. S. 1979, PASP, 91, 589

Blain, A. W., Smail, I., Ivison, R. J., \& Kneib, J.-P. 1999, MNRAS, 302,632

Bravo-Alfaro, H., Brinks, E., Baker, A. J., Walter, F., \& Kunth, D. 2004, AJ, 127, 264

Bressan, A., Silva, L., \& Granato, G. L. 2002, A\&A, 392, 377

Calzetti, D., Meurer, G. R., Bohlin, R. C., et al. 1997, AJ, 114, 1834

Cannon, J. M., \& Skillman, E. D. 2004, ApJ, 610, 772

Cardelli, J. A., Clayton, G. C., \& Mathis, J. S. 1989, ApJ, 345, 245

Carilli, C. L., \& Yun, M. S. 1999, ApJ, 513, L13

Chapman, S. C., Windhorst, R., Odewahn, S., Yan, H., \& Conselice, C. 2003, ApJ, 599, 92

Chary, R., \& Elbaz, D. 2001, ApJ, 556, 562

Cole, S., Lacey, C. G., Baugh, C. M., \& Frenk, C. S. 2000, MNRAS, 319, 168

Condon, J. J. 1992, ARA\&A, 30, 575

Condon, J. J., Anderson, M. L., \& Helou, G. 1991, ApJ, 376, 95

Conti, P. S. 1991, ApJ, 377, 115

Cox, A. L., Sparke, L. S., Watson, A. M., \& van Moorsel, G. 2001, AJ, 121, 692

Dale, D. A., Helou, G., Contursi, A., Silbermann, N. A., \& Kolhatkar, S. 2001a, ApJ, 549, 215
Dale, D. A., Helou, G., Neugebauer, G., Soifer, B. T., Frayer, D. T., \& Condon, J. J. 2001b, AJ, 122, 1736

Dale, D. A., \& Helou, G. 2002, ApJ, 576, 159

Dannerbauer, H., Lehnert, M. D., Lutz, D., et al. 2004, ApJ, 606, 664

de Jong, R. S. 1996, A\&A, 313, 377

de Grijs, R., et al. 2004, MNRAS, 352, 263

Desert, F.-X., Boulanger, F., \& Puget, J. L. 1990, A\&A, 237, 215

Draine, B. T., \& Lee, H. M. 1984, ApJ, 285, 89

Dunne, L., Eales, S., Edmunds, M., et al. 2000, 315, 115

Dwek, E., \& Scalo, J. M. 1980, ApJ, 239, 193

Edmunds, M. G. 2001, MNRAS, 328, 223

Förster Schreiber, N. M., Roussel, H., Sauvage, M., \& Charmandaris, V. 2004, A\&A, 419, 501

Gallagher, J. S., \& Smith, L. J. 1999, MNRAS, 304, 540

Garrett, M. A. 2002, A\&A, 384, L19

Genzel, R., Baker, A. J., Tacconi, L. J., et al. 2003, ApJ, 584, 633

Gil de Paz, A., Madore, B. F., \& Pevunova, O. 2003, ApJS, 147, 29

Glass, I. S., \& Moorwood, A. F. M. 1985, MNRAS, 214, 429

Gorjian, V., Turner, J. L., \& Beck, S. C. 2001, ApJ, 554, L29

Guseva, N. G., Izotov, Y. I., \& Thuan, T. X. 2000, ApJ, 531, 776

Haarsma, D. B., Partridge, R. B., Windhorst, R. A., \& Richards, E. A. 2000, ApJ, 544, 641

Haas, M., Klaas, U., \& Bianchi, S. 2002, A\&A, 385, L23

Hanner, M. S. 1988, NASA Conf. Pub., 3004, 22

Hawarden, T. G., Leggett, S. K., Letawsky, M. B., Ballantyne, D. R., \& Casali, M. M. 2001, MNRAS, 325, 563

Helou, G., Soifer, B. T., \& Rowan-Robinson, M. 1985, ApJ, 298, L7

Hildebrand, R. H. 1983, Royal Astron. Soc. Quart., 24, 267

Hirashita, H., \& Hunt, L. K. 2004, A\&A, 421, 555

Hirashita, H., Hunt, L. K., \& Ferrara, A. 2002a, MNRAS, 330, L19

Hirashita, H., Tajiri, Y. Y., \& Kamaya, H. 2002b, A\&A, 388, 439

Ho, P. T. P., Beck, S. C., \& Turner, J. L. 1990, ApJ, 349, 57

Hopkins, A. M., Schulte-Ladbeck, R. E., \& Drozdovsky, I. O. 2002, AJ, 124, 862

Houck, J. R., Charmandaris, V., Brandl, B. R., et al. 2004, ApJS, Spitzer Special Issue

Hughes, D. H., Aretxaga, I., Chapin, E. L., et al. 2002, MNRAS, 335, 871

Hunt, L. K., Vanzi, L., \& Thuan, T. X. 2001, A\&A, 377, 66

Hunt, L. K., Giovanardi, C., \& Helou, G. 2002, A\&A, 394, 873

Hunt, L. K., Thuan, T. X., \& Izotov, Y. I. 2003, ApJ, 588, 281

Hunt, L. K., Dyer, K. K., Thuan, T. X., \& Ulvestad, J. S. 2004, ApJ, 606,853

Issa, M. R., MacLaren, I., \& Wolfendale, A. W. 1990, A\&A, 236, 237

Ivezić, Z., \& Elitzur, M. 1997, MNRAS, 287, 799

Ivezić, Z., Nenkova, M., \& Elitzur, M. 1999, User Manual for DUSTY, University of Kentucky Internal Report, accessible at http://www. pa.uky. edu/ moshe/dusty

Izotov, Y. I., \& Thuan, T. X. 1999, ApJ, 511, 639

James, A., Dunne, L., Eales, S., \& Edmunds, M. G. 2002, MNRAS, 335,753

Jarrett, T. H., Chester, T., Cutri, R., Schneider, S. E., \& Huchra, J. P. 2003, AJ, 125, 525

Jenness, T., \& Lightfoot, J. F. 1998, SURF - SCUBA User Reduction Facility, Starlink User Note 216, Starlink Project, CLRC

Jenness, T., Stevens, J. A., Archibald, E. N., et al. 2002, MNRAS, 336, 14

Johnson, K. E., Leitherer, C., Vacca, W. D., \& Conti, P. S. 2000, AJ, 120,1273

Joy, M., \& Lester, D. F. 1988, ApJ, 331, 145

Kawara, K., Nishida, M., \& Phillips, M. M. 1989, ApJ, 337, 230

Kennicutt, R. C. 1998, ARA\&A, 36, 189

Kim, S., Martin, P. G., \& Hendry, P. D. 1994, ApJ, 422, 164 
Klein, U., Weiland, H., \& Brinks, E. 1991, A\&A, 246, 323

Kobulnicky, H. A., \& Johnson, K. E. 1999, ApJ, 527, 154

Kobulnicky, H. A., Dickey, J. M., Sargent, A. I., Hogg, D. E., \& Conti, P. S. 1995, AJ, 110, 116

Kobulnicky, H. A., Kennicutt, R. C., Jr., \& Pizagno, J. L. 1999, ApJ, 514,544

Kobulnicky, H. A., \& Skillman, E. D. 1995, ApJ, 454, L121

Kobulnicky, H. A., \& Skillmann, E. D. 1996, ApJ, 471, 211

Kunth, D., \& Östlin, G. 2000, A\&AR, 10, 1

Legrand, F., Kunth, D., Mas-Hesse, J. M., \& Lequeux, J. 1997, A\&A, 326, 929

Leitherer, C., Schaerer, D., Goldader, J. D., et al. 1999, ApJS, 123, 3

Lisenfeld, U., \& Ferrara, A. 1998, ApJ, 496, 145

Madau, P., Ferguson, H. C., Dickinson, M. E., et al. 1996, MNRAS, 283,1388

Maiolino, R., Schneider, R., Oliva, E., et al. 2004, Nature, 431, 533

Marchenko, S. V., Moffat, A. F. J., Vacca, W. D., Côté, S., \& Doyon, R. 2002, ApJ, 565, L59

Marlowe, A. T., Meurer, G. R., Heckman, T. M., \& Schommer, R. 1997, ApJS, 112, 285

Martin, C. L. 1996, ApJ, 465, 680

Martin, C. L. 1997, ApJ, 491, 561

Mathis, J. S., Rumpl, W., \& Nordsieck, K. H. 1977, ApJ, 217, 425

Meier, D. S., Turner, J. L., Crosthwaite, L. P., \& Beck, S. C. 2001, AJ, 121,740

Meier, D. S., Turner, J. L., \& Beck, S. C. 2002, AJ, 124, 877

Mendez, D. I., \& Esteban, C. 1997, ApJ, 488, 652

Moorwood, A. F. M., \& Glass, I. S. 1982, A\&A, 115, 84

Morgan, H. L., \& Edmunds, M. G. 2003, MNRAS, 343, 427

Östlin, G. 2000, ApJ, 535, L99

Pettini, M., Shapley, A. E., Steidel, C. C., et al. 2001, ApJ, 554, 981

Phillips, M. M., Aitken, D. K., \& Roche, P. F. 1984, MNRAS, 207, 25

Plante, S., \& Sauvage, M. 2002, AJ, 124, 1995

Price, R., \& Duric, N. 1992, ApJ, 401, 81

Puget, J. L., \& Leger, A. 1989, ARA\&A, 27, 161

Rees, M. J. 1998, Space Science Rev., 84, 43

Rigopoulou, D., Kunze, D., Lutz, D., Genzel, R., \& Moorwood, A. F. M. 2002, A\&A, 389, 374

Roche, P. F., Aitken, D. K., Smith, C. H., \& Ward, M. J. 1991, MNRAS, 248, 606

Roussel, H., Sauvage, M., Vigroux, L., \& Bosma, A. 2001, A\&A, 372, 427

Roussel, H., Helou, G., Beck, R., et al. 2003, ApJ, 593, 733

Rowan-Robinson, M. 1980, ApJS, 44, 403
Rowan-Robinson, M., Mann, R. G., Oliver, S. J., et al. 1997, MNRAS, 289,490

Sandage, A., Saha, A., Tammann, G. A., et al. 1994, ApJ, 423, L13

Schaerer, D., \& Vacca, W. D. 1998, ApJ, 497, 618

Schaerer, D., Contini, T., Kunth, D., \& Meynet, G. 1997, ApJ, 481, L75

Schlegel, D. J., Finkbeiner, D. P., \& Davis, M. 1998, ApJ, 500, 525

Silva, L., Granato, G. L., Bressan, A., \& Danese, L. 1998, ApJ, 509, 103

Shapley, A. E., Erb, D. K., Pettini, M., Steidel, C. C., \& Adelberger, K. L. 2004, ApJ, 612, 108

Smith, L. J., \& Gallagher, J. S. 2001, MNRAS, 326, 1027

Spitzer, L., Jr. 1978, Physical Processes in the Interstellar Medium (New York: John Wiley \& Sons)

Summers, L. K., Stevens, I. R., \& Strickland, D. K. 2001, MNRAS, 327,385

Takagi, T., Arimoto, N., \& Hanami, H. 2003, MNRAS, 340, 813

Takeuchi, T. T., Hirashita, H., Ishii, T. T., Hunt, L. K., \& Ferrara, A. 2003, MNRAS, 343, 839

Taylor, C. L., Kobulnicky, H. A., \& Skillman, E. D. 1998, AJ, 116, 2746

Tecza, M., Baker, A. J., Davies, R. I., et al. 2004, ApJ, 605, L109

Thuan, T. X., Izotov, Y. I., \& Lipovetsky, V. A. 1997, ApJ, 477, 661

Thuan, T. X., Lipovetsky, V. A., Martin, J.-M., \& Pustilnik, S. A. 1999a, A\&AS, 139, 1

Thuan, T. X., Sauvage, M., \& Madden, S. 1999b, ApJ, 516, 783

Todini, P., \& Ferrara, A. 2001, MNRAS, 325, 726

Tokunaga, A. T. 1984, AJ, 89, 172

Turner, J. L., Beck, S. C., \& Ho, P. T. P. 2000, ApJ, 532, L109

Vacca, W. D., Johnson, K. E., \& Conti, P. S. 2002, AJ, 123, 772

Vader, J. P., Frogel, J. A., Terndrup, D. M., \& Heisler, C. A. 1993, AJ, 106,1743

Vanzi, L., Rieke, G. H., Martin, C. L., \& Shields, J. C. 1996, ApJ, 466, 150

Vanzi, L., \& Sauvage, M. 2004, A\&A, 415, 509

Verma, A., Lutz, D., Sturm, E., et al. 2003, A\&A, 403, 829

Walsh, J. R., \& Roy, J. 1993, MNRAS, 262, 27

Weingartner, J. C., \& Draine, B. T. 2001, ApJ, 548, 296

Wood, D. O. S., \& Churchwell, E. 1989, ApJS, 69, 831

Wynn-Williams, C. G., \& Becklin, E. E. 1986, ApJ, 308, 620

Yun, M. S., \& Carilli, C. L. 2002, ApJ, 568, 88

van Zee, L., Skillman, E. D., \& Salzer, J. J. 1998, AJ, 116, 1186

Zubko, V. G., Mennella, V., Colangeli, L., \& Bussoletti, E. 1996, MNRAS, 282, 1321 


\section{Online Material}


L. Hunt et al.: SEDs of low-metallicity BCDs, Online Material p 2

\section{Appendix A: SED data and references}

The fluxes used in the SEDs together with their references are given in Tables A1 through A7 for He 2-10, II Zw 40, II Zw 70, I Zw 18, Mrk 33, NGC 5253, and SBS 0335-052, respectively. Wavelengths in the tables are given as $\mu \mathrm{m}$ up to the "Radio" region, where they are given in $\mathrm{cm}$. The values reported here have not been corrected for Galactic extinction. The crossidentification of the data references follow the individual data tables.

\section{Data references}

[A1] Johansson, I. 1987, A\&A, 182, 179

[A2] NASA Extragalactic Database (NED)

[A3] Noeske, K. G., Papaderos, P., Cairós, L. M., \& Fricke, K. J. 2003, A\&A, 410, 481

[A4] Telesco, C. M., Dressel, L. L., \& Wolstencroft, R. D. 1993, ApJ, 414, 120

[A5] Vacca, W. D., Johnson, K. E., \& Conti, P. S. 2002, AJ, 123,772

[A6] Sauvage, M., Thuan, T. X., \& Lagage, P. O. 1997, A\&A, 325, 98

[A7] Beck, S. C., Turner, J. L., \& Gorjian, V. 2001, AJ, 122, 1365

\section{[A8] ISSA/OASIS}

[A9] Kobulnicky, H. A., \& Johnson, K. E. 1999, ApJ, 527, 154

[A10] Marlowe, A. T., Meurer, G. R., Heckman, T. M., \& Schommer, R. 1997, ApJS, 112

[A11] Heisler, C. A., \& Vader, J. P. 1994, AJ, 107, 35

[A12] Telles, E., \& Terlevich, R. 1997, MNRAS, 286, 183

[A13] Beck, S. C., Turner, J. L., Langland-Shula, L. E., et al. 2002, AJ, 124, 2516

[A14] Vader, J. P., Frogel, J. A., Terndrup, D. M., \& Heisler, C. A. 1993, AJ, 106, 1743

[A15] Albrecht, M., Chini, R., Krügel, E., Müller, S. A. H., \& Lemke, R. 2004, A\&A, 414, 141

[A16] Klein, U., Weiland, H., \& Brinks, E. 1991, A\&A, 246, 323

[A17] Deeg, H., Brinks, E., Duric, N., Klein, U., \& Skillman, E. 1993, ApJ, 410, 626

[A18] Condon, J. J., Cotton, W. D., Greisen, E. W., et al. 1998, AJ, 115, 1693: National Radio Astronomy Observatory Very Large Array Sky Survey (NVSS)

[A19] Gil de Paz, A., Madore, B. F., \& Pevunova, O. 2003, ApJS, 147, 29

[A20] Cox, A. L., Sparke, L. S., Watson, A. M., \& van Moorsel, G. 2001, AJ, 121, 692

[A21] Hunt, L. K., Thuan, T. X., \& Izotov, Y. I. 2003, ApJ, 588,281

[A22] Hunt, L. K., Dyer, K., \& Thuan, T. X. 2004, in preparation

[A23] Lequeux, J., \& Viallefond, F. 1980, A\&A, 91, 269

[A24] Summers, L. K., Stevens, I. R., \& Strickland, D. K. 2001, MNRAS, 327, 385

[A25] Huchra, J. P. 1977, ApJS, 35, 171

[A26] Beck, S. C., Turner, J. L., \& Kovo, O. 2000, AJ, 120, 244
Table A1. SED for He $2-10$

\begin{tabular}{|c|c|c|c|c|}
\hline Region & $\lambda(\mu \mathrm{m})$ & Flux (mJy) & Error (mJy) & Ref. \\
\hline OPT+NIR & 0.36 & 19.66 & 1.81 & $\mathrm{~A} 1\left(61^{\prime \prime}\right)$ \\
\hline $\mathrm{OPT}+\mathrm{NIR}$ & 0.44 & 35.76 & 3.29 & $\mathrm{~A} 1\left(61^{\prime \prime}\right)$ \\
\hline $\mathrm{OPT}+\mathrm{NIR}$ & 0.44 & 44.61 & 3.70 & A2 \\
\hline $\mathrm{OPT}+\mathrm{NIR}$ & 0.55 & 50.25 & 4.63 & $\mathrm{~A} 1\left(61^{\prime \prime}\right)$ \\
\hline $\mathrm{OPT}+\mathrm{NIR}$ & 0.64 & 112.86 & 9.35 & A2 \\
\hline $\mathrm{OPT}+\mathrm{NIR}$ & 0.64 & 63.76 & 5.87 & $\mathrm{~A} 1\left(61^{\prime \prime}\right)$ \\
\hline $\mathrm{OPT}+\mathrm{NIR}$ & 0.79 & 77.72 & 7.16 & $\mathrm{~A} 1\left(61^{\prime \prime}\right)$ \\
\hline $\mathrm{OPT}+\mathrm{NIR}$ & 1.2 & 103.78 & 9.56 & $\mathrm{~A} 1\left(30^{\prime \prime}\right)$ \\
\hline $\mathrm{OPT}+\mathrm{NIR}$ & 1.2 & 178.53 & 2.30 & $\mathrm{~A} 2$ \\
\hline $\mathrm{OPT}+\mathrm{NIR}$ & 1.2 & 210.92 & 19.42 & A3 \\
\hline $\mathrm{OPT}+\mathrm{NIR}$ & 1.6 & 126.07 & 11.61 & $\mathrm{~A} 1\left(30^{\prime \prime}\right)$ \\
\hline $\mathrm{OPT}+\mathrm{NIR}$ & 1.6 & 183.57 & 2.87 & A2 \\
\hline $\mathrm{OPT}+\mathrm{NIR}$ & 1.6 & 227.30 & 20.93 & A3 \\
\hline $\mathrm{OPT}+\mathrm{NIR}$ & 2.2 & 104.13 & 9.59 & $\mathrm{~A} 1\left(30^{\prime \prime}\right)$ \\
\hline $\mathrm{OPT}+\mathrm{NIR}$ & 2.2 & 164.42 & 3.63 & A2 \\
\hline $\mathrm{OPT}+\mathrm{NIR}$ & 2.2 & 182.63 & 16.82 & A3 \\
\hline MIR & 10.8 & 600.0 & 40.0 & $\mathrm{~A} 4$ \\
\hline MIR & 10.8 & 720.0 & 95.0 & A5 \\
\hline MIR & 11.7 & 850.0 & 20.0 & A6 \\
\hline MIR & 11.7 & 880.0 & 9.0 & A7 \\
\hline MIR & 12.0 & 1093.0 & 109.0 & A8 \\
\hline MIR & 25.0 & 6511.0 & 651.0 & A 8 \\
\hline FIR-mm & 60 & 24080.0 & 2408.0 & A8 \\
\hline FIR-mm & 100 & 26400.0 & 2640.0 & A8 \\
\hline FIR-mm & 450 & 580.0 & 150.0 & This paper \\
\hline FIR-mm & 850 & 140.0 & 15.0 & This paper \\
\hline FIR-mm & 0.1 & 5.9 & 3.30 & A9 \\
\hline FIR-mm & 0.3 & 6.2 & 0.60 & A9 \\
\hline Radio & 2.0 & 10.9 & 1.00 & A7 \\
\hline Radio & 2.0 & 21.1 & 1.20 & A9 \\
\hline Radio & 2.8 & 40.0 & 15.00 & A9 \\
\hline Radio & 3.4 & 35.0 & 8.00 & A9 \\
\hline Radio & 3.6 & 24.3 & 2.40 & A9 \\
\hline Radio & 3.6 & 29.0 & 3.00 & A9 \\
\hline Radio & 4.8 & 45.0 & 10.00 & A9 \\
\hline Radio & 6.0 & 55.0 & 10.00 & A9 \\
\hline Radio & 6.2 & 42.6 & 4.30 & A9 \\
\hline Radio & 11.1 & 75.0 & 35.00 & A9 \\
\hline Radio & 21.4 & 69.2 & 6.90 & A9 \\
\hline Radio & 73.5 & 240.0 & 80.00 & A9 \\
\hline Radio & 92.2 & 100.0 & 20.00 & A9 \\
\hline
\end{tabular}

[A27] Moorwood, A. F. M., \& Glass, I. S. 1982, A\&A, 115, 84

[A28] Jarrett, T. H., Chester, T., Cutri, R., Schneider, S. E., \& Huchra, J. P. 2003, AJ, 125, 525

[A29] Vanzi, L., \& Sauvage, M. 2004, A\&A, 415, 509

[A30] Frogel, J. F., Elias, J. H., \& Phillips, M. M. 1982, ApJ, 260,70

[A31] Gorjian, V., Turner, J. L., \& Beck, S. C. 2001, ApJ, 554, L29

[A32] Beck, S. C., Turner, J. L., Ho, P. T. P., Lacy, J. H., \& Kelly, D. M. 1996, ApJ, 457, 610

[A33] Mohan, N. R., Anantharamaiah, K. R., \& Goss, W. M. 2001, ApJ, 557, 659

[A34] Schmitt, H. R., Kinney, A. L., Calzetti, D., \& Storchi Bergmann, T. 1997, AJ, 114, 592

[A35] Papaderos, P., Izotov, Y. I., Fricke, K. J., Thuan, T. X., \& Guseva, N. G. 1998, A\&A, 338, 43

[A36] Thuan, T. X., Izotov, Y. I., \& Lipovetsky, V. A. 1997, ApJ, 477, 661

[A37] Vanzi, L., Hunt, L. K., Thuan, T. X., \& Izotov, Y. I. 2000, A\&A, 363, 493

[A38] Hunt, L. K., Vanzi, L., \& Thuan, T. X. 2001, A\&A, 377, 66 
L. Hunt et al.: SEDs of low-metallicity BCDs, Online Material p 3

Table A2. SED for II Zw 40

\begin{tabular}{|c|c|c|c|c|}
\hline Region & $\lambda(\mu \mathrm{m})$ & Flux (mJy) & Error (mJy) & Ref. \\
\hline OPT+NIR & 0.36 & 1.91 & 0.07 & A10 $\left(166^{\prime \prime}\right)$ \\
\hline $\mathrm{OPT}+\mathrm{NIR}$ & 0.36 & 0.47 & 0.04 & A11 \\
\hline $\mathrm{OPT}+\mathrm{NIR}$ & 0.43 & 3.29 & 0.09 & $\mathrm{~A} 10\left(166^{\prime \prime}\right)$ \\
\hline $\mathrm{OPT}+\mathrm{NIR}$ & 0.44 & 1.58 & 0.15 & A11 \\
\hline $\mathrm{OPT}+\mathrm{NIR}$ & 0.44 & 2.74 & 0.33 & A2 \\
\hline $\mathrm{OPT}+\mathrm{NIR}$ & 0.54 & 6.94 & 0.13 & $\operatorname{A} 10\left(166^{\prime \prime}\right)$ \\
\hline $\mathrm{OPT}+\mathrm{NIR}$ & 0.55 & 4.98 & 0.60 & A2 \\
\hline $\mathrm{OPT}+\mathrm{NIR}$ & 0.55 & 5.31 & 0.10 & A12 \\
\hline $\mathrm{OPT}+\mathrm{NIR}$ & 0.55 & 5.77 & 0.53 & A11 \\
\hline $\mathrm{OPT}+\mathrm{NIR}$ & 0.64 & 6.99 & 0.64 & A11 \\
\hline $\mathrm{OPT}+\mathrm{NIR}$ & 0.64 & 8.56 & 0.16 & A12 \\
\hline $\mathrm{OPT}+\mathrm{NIR}$ & 0.79 & 10.06 & 0.93 & A11 \\
\hline $\mathrm{OPT}+\mathrm{NIR}$ & 0.79 & 8.76 & 0.16 & A12 \\
\hline $\mathrm{OPT}+\mathrm{NIR}$ & 0.82 & 12.22 & 0.34 & $\mathrm{~A} 10\left(166^{\prime \prime}\right)$ \\
\hline $\mathrm{OPT}+\mathrm{NIR}$ & 1.2 & 8.40 & 0.31 & This paper $\left(20^{\prime \prime}\right)$ \\
\hline $\mathrm{OPT}+\mathrm{NIR}$ & 1.2 & 8.63 & 0.44 & A2 \\
\hline $\mathrm{OPT}+\mathrm{NIR}$ & 1.6 & 9.13 & 0.34 & This paper $\left(20^{\prime \prime}\right)$ \\
\hline $\mathrm{OPT}+\mathrm{NIR}$ & 1.6 & 10.01 & 0.68 & A2 \\
\hline $\mathrm{OPT}+\mathrm{NIR}$ & 2.2 & 7.54 & 0.65 & This paper $\left(20^{\prime \prime}\right)$ \\
\hline $\mathrm{OPT}+\mathrm{NIR}$ & 2.2 & 8.99 & 0.33 & $\mathrm{~A} 2$ \\
\hline MIR & 7.0 & 50.0 & 50.0 & This paper (ISO archive) \\
\hline MIR & 8.0 & 80.0 & 50.0 & This paper (ISO archive) \\
\hline MIR & 10.0 & 120.0 & 50.0 & This paper (ISO archive) \\
\hline MIR & 10.1 & 200.0 & 20.0 & A13 \\
\hline MIR & 10.4 & 220.0 & 40.0 & A13 \\
\hline MIR & 11.7 & 240.0 & 40.0 & A13 \\
\hline MIR & 12.0 & 300.0 & 50.0 & This paper (ISO archive) \\
\hline MIR & 12.0 & 460.0 & 50.0 & A14 \\
\hline MIR & 13.0 & 350.0 & 50.0 & This paper (ISO archive) \\
\hline MIR & 14.0 & 400.0 & 50.0 & This paper (ISO archive) \\
\hline MIR & 15.0 & 530.0 & 50.0 & This paper (ISO archive) \\
\hline MIR & 20.0 & 1000.0 & 200.0 & A13 \\
\hline MIR & 25.0 & 1910.0 & 200.0 & A14 \\
\hline FIR-mm & 60 & 6610.0 & 700.0 & A14 \\
\hline FIR-mm & 100 & 5800.0 & 900.0 & A14 \\
\hline FIR-mm & 450 & 400.0 & 90.0 & This paper \\
\hline FIR-mm & 850 & 50.0 & 10.0 & This paper $\left(40^{\prime \prime}\right)$ \\
\hline FIR-mm & 850 & 90.0 & 10.0 & This paper (total) \\
\hline FIR-mm & 1300 & 13.3 & 3.1 & A15 \\
\hline Radio & 1.2 & 18.0 & 4.00 & A16 \\
\hline Radio & 2.0 & 12.0 & 3.00 & A16 \\
\hline Radio & 2.8 & 20.0 & 1.00 & A16 \\
\hline Radio & 6.0 & 22.0 & 0.10 & A16 \\
\hline Radio & 20.1 & 30.5 & 1.50 & A17 \\
\hline Radio & 20.7 & 30.0 & 0.50 & A16 \\
\hline Radio & 21.4 & 34.2 & 1.40 & A18 \\
\hline Radio & 92.2 & 38.0 & 4.00 & $\mathrm{~A} 17$ \\
\hline
\end{tabular}

[A39] Houck, J. R., et al. 2004, ApJS, 154, 211

[A40] Thuan, T. X., Sauvage, M., \& Madden, S. 1999, ApJ, 516,783

[A41] Plante, S., \& Sauvage, M. 2002, AJ, 124, 1995

[A42] Dale, D. A., Helou, G., Neugebauer, G., Soifer, B. T., Frayer, D. T., \& Condon, J. J. 2001, AJ, 122, 1736

[A43] Hunt, L. K., Dyer, K. K., Thuan, T. X., \& Ulvestad, J. S. 2004, ApJ, 606, 853

\section{Appendix B: Dust mass from DUSTY}

Here we set out the equations used to infer the dust mass from the output given by DUSTY. Assuming that the dust is distributed in a spherical shell with a power-law radial density profile $\rho(r)=C_{1} r^{-p}$, the dust mass can be written as:

$$
\begin{aligned}
M_{\mathrm{dust}} & =\int_{R_{\text {in }}}^{R_{\text {out }}} 4 \pi r^{2} C_{1} r^{-p} \mathrm{~d} r \\
& =4 \pi C_{1} \begin{cases}\frac{R_{\text {out }}^{3-p}-R_{\text {in }}^{3-p}}{3-p} & p \neq 3 \\
\ln \frac{R_{\text {out }}}{R_{\text {in }}} & p=3\end{cases}
\end{aligned}
$$

where $C_{1}$ is a constant, and $R_{\text {in }}$ and $R_{\text {out }}$ are the inner and outer shell radii.
Table A3. SED for II Zw 70

\begin{tabular}{lrrrl} 
Region & $\lambda(\mu \mathrm{m})$ & Flux $(\mathrm{mJy})$ & Error $(\mathrm{mJy})$ & \multicolumn{1}{c}{ Ref. } \\
\hline OPT+NIR & 0.44 & 4.94 & 0.55 & $\mathrm{~A} 19$ \\
OPT+NIR & 0.44 & 4.98 & 0.60 & $\mathrm{~A} 2$ \\
OPT+NIR & 0.44 & 5.22 & 0.48 & $\mathrm{~A} 20$ \\
OPT+NIR & 0.55 & 5.41 & 0.65 & $\mathrm{~A} 2$ \\
OPT+NIR & 0.55 & 6.04 & 0.56 & $\mathrm{~A} 20$ \\
OPT+NIR & 0.64 & 5.92 & 0.71 & $\mathrm{~A} 20$ \\
OPT+NIR & 0.64 & 6.26 & 0.58 & $\mathrm{~A} 19$ \\
OPT+NIR & 1.2 & 5.81 & 0.32 & $\mathrm{~A} 2$ \\
OPT+NIR & 1.6 & 6.03 & 0.44 & $\mathrm{~A} 2$ \\
OPT+NIR & 2.2 & 4.98 & 0.41 & $\mathrm{~A} 2$ \\
FIR-mm & 25.0 & 133.0 & 26.6 & $\mathrm{~A} 2$ \\
FIR-mm & 60 & 714.0 & 50.0 & $\mathrm{~A} 2$ \\
FIR-mm & 100 & 1237.0 & 123.7 & $\mathrm{~A} 2$ \\
FIR-mm & 850 & 20.0 & $\mathrm{UL}$ & $\mathrm{This}$ paper \\
Radio & 2.8 & 2.7 & 0.20 & $\mathrm{~A} 17$ \\
Radio & 2.8 & 4.1 & 2.00 & $\mathrm{~A} 17$ \\
Radio & 2.8 & 4.4 & 1.00 & $\mathrm{~A} 17$ \\
Radio & 6.1 & 3.0 & 1.00 & $\mathrm{~A} 17$ \\
Radio & 6.3 & 2.5 & 1.10 & $\mathrm{~A} 17$ \\
Radio & 6.3 & 3.4 & 1.00 & $\mathrm{~A} 17$ \\
Radio & 20.5 & 4.3 & 1.00 & $\mathrm{~A} 17$ \\
Radio & 21.2 & 5.0 & 0.60 & $\mathrm{~A} 17$ \\
Radio & 49.1 & 6.5 & 0.80 & $\mathrm{~A} 17$ \\
Radio & 91.7 & 11.2 & 2.00 & $\mathrm{~A} 17$ \\
Radio & 92.2 & 12.5 & 3.00 & $\mathrm{~A} 17$ \\
\hline & & & &
\end{tabular}

Table A4. SED for IZw 18

\begin{tabular}{lrrrl}
\multicolumn{1}{c}{ Region } & $\lambda(\mu \mathrm{m})$ & Flux $(\mathrm{mJy})$ & Error $(\mathrm{mJy})$ & \multicolumn{1}{c}{ Ref. } \\
\hline OPT+NIR & 0.439 & 1.10 & 0.05 & $\mathrm{~A} 21\left(20^{\prime \prime}\right)$ \\
OPT+NIR & 0.44 & 1.62 & 0.06 & $\mathrm{~A} 19$ \\
OPT+NIR & 0.555 & 1.19 & 0.05 & $\mathrm{~A} 21\left(20^{\prime \prime}\right)$ \\
OPT+NIR & 0.64 & 0.98 & 0.06 & $\mathrm{~A} 19$ \\
OPT+NIR & 0.814 & 0.76 & 0.03 & $\mathrm{~A} 21\left(20^{\prime \prime}\right)$ \\
OPT+NIR & 1.2 & 0.76 & 0.03 & $\mathrm{~A} 21\left(20^{\prime \prime}\right)$ \\
OPT+NIR & 1.6 & 0.64 & 0.02 & $\mathrm{~A} 21\left(20^{\prime \prime}\right)$ \\
OPT+NIR & 2.2 & 0.51 & 0.02 & $\mathrm{~A} 21\left(20^{\prime \prime}\right)$ \\
FIR-mm & 850 & 2.5 & $\mathrm{UL}$ & This paper \\
FIR-mm & 1200 & 5.5 & $\mathrm{UL}$ & F. Bertoldi, priv. comm. \\
Radio & 2.8 & 2.1 & 0.60 & $\mathrm{~A} 16$ \\
Radio & 3.5 & 0.6 & 0.04 & $\mathrm{~A} 22$ \\
Radio & 3.5 & 0.8 & 0.09 & $\mathrm{~A} 22$ \\
Radio & 6.0 & 1.6 & 0.20 & $\mathrm{~A} 23$ \\
Radio & 6.2 & 1.0 & 0.18 & $\mathrm{~A} 22$ \\
Radio & 6.2 & 0.5 & 0.07 & $\mathrm{~A} 22$ \\
Radio & 6.3 & 1.6 & 0.20 & $\mathrm{~A} 16$ \\
Radio & 21.0 & 1.2 & 0.19 & $\mathrm{~A} 22$ \\
Radio & 21.0 & 1.6 & 0.15 & $\mathrm{~A} 22$ \\
Radio & 21.0 & 2.6 & 0.90 & $\mathrm{~A} 16$ \\
\hline
\end{tabular}

DUSTY provides the value of $\tau_{v}$, the optical depth at frequency $v$, which can be expressed as:

$$
\begin{aligned}
\tau_{v} & =\int_{R_{\text {in }}}^{R_{\text {out }}} \mathcal{X}_{v}(r) \mathrm{d} r \\
& =\int_{R_{\text {in }}}^{R_{\text {out }}} \mathrm{d} r \int_{a_{1}}^{a_{2}} \mathrm{~d} a \pi a^{2}\left[\sum_{i} Q_{i, v}(a) n_{i}(a, r)\right]
\end{aligned}
$$

where $i$ refers to the grain chemical composition type. $\mathcal{X}_{v}$ is the absorption coefficient per unit length; $Q_{i, v}(a)$ the extinction efficiency factor (ratio of optical to geometrical cross section) for type $i$ grains with size (radius) $a ; n_{i}(a, r)$ the number density distribution in radius for grains of type $i$ at radius $r$ of size (radius) $a$; and $\pi a^{2}$ the geometrical cross section of a grain with size (radius) $a . a_{1}$ and $a_{2}$ are the minimum and maximum grain sizes, respectively. $n_{i}(a, r)$ can be written as two functions which separate the mass distribution radial dependence from the grain size: $n_{i}(a, r)=N_{i}(r) \mathcal{F}(a)$. Assuming the grain sizes are distributed as a power law, $\mathcal{F}(a)=A a^{-q}$, where $A$ is 
L. Hunt et al.: SEDs of low-metallicity BCDs, Online Material p 4

Table A5. SED for Mrk 33

\begin{tabular}{lrrrl} 
Region & $\lambda(\mu \mathrm{m})$ & Flux $(\mathrm{mJy})$ & Error $(\mathrm{mJy})$ & \multicolumn{1}{c}{ Ref. } \\
\hline OPT+NIR & 0.44 & 16.96 & 1.56 & $\mathrm{~A} 2$ \\
OPT+NIR & 0.44 & 18.60 & 3.42 & $\mathrm{~A} 24$ \\
OPT+NIR & 0.44 & 18.77 & 0.69 & $\mathrm{~A} 19$ \\
OPT+NIR & 0.55 & 13.46 & 0.62 & $\mathrm{~A} 25$ \\
OPT+NIR & 0.55 & 17.42 & 0.80 & $\mathrm{~A} 25$ \\
OPT+NIR & 0.64 & 21.91 & 2.22 & $\mathrm{~A} 19$ \\
OPT+NIR & 0.64 & 28.09 & 2.59 & $\mathrm{~A} 24$ \\
OPT+NIR & 1.2 & 43.66 & 1.21 & $\mathrm{~A} 2$ \\
OPT+NIR & 1.2 & 47.44 & 4.37 & This paper \\
OPT+NIR & 1.6 & 52.55 & 4.84 & This paper \\
OPT+NIR & 1.6 & 53.53 & 1.73 & A2 \\
OPT+NIR & 2.2 & 39.59 & 3.65 & This paper \\
OPT+NIR & 2.2 & 44.22 & 1.63 & A2 \\
MIR & 12.0 & 210.0 & 31.0 & A2 \\
MIR & 25.0 & 1050.0 & 30.0 & A2 \\
FIR-mm & 60 & 4770.0 & 42.0 & A2 \\
FIR-mm & 100 & 5990.0 & 129.0 & A2 \\
FIR-mm & 450 & 170.0 & UL & This paper \\
FIR-mm & 850 & 50.0 & 10.0 & This paper \\
Radio & 1.2 & 10.0 & 3.00 & A16 \\
Radio & 1.3 & 3.2 & 1.80 & A16 \\
Radio & 2.0 & 16.0 & 0.06 & A26 \\
Radio & 2.8 & 7.1 & 0.90 & A16 \\
Radio & 3.6 & 4.9 & 0.05 & A26 \\
Radio & 6.0 & 11.0 & 0.05 & A26 \\
Radio & 6.1 & 31.0 & 6.00 & A16 \\
Radio & 6.3 & 12.0 & 1.00 & A16 \\
Radio & 11.1 & 24.0 & 6.00 & A16 \\
Radio & 20.0 & 20.0 & 0.08 & A26 \\
& & & &
\end{tabular}

such that $\int_{a_{1}}^{a_{2}} \mathcal{F}(a) \mathrm{d} a=1 . N_{i}(r)$ is the number density of grain type $i$ at radius $r$.

We can now write $\rho_{i}$ the mass density at radius $r$ of grain type $i$ as:

$$
\begin{aligned}
\rho_{i}(r) & =\int_{a_{1}}^{a_{2}} \delta_{i}\left(\frac{4}{3} \pi a^{3}\right) n_{i}(a, r) \mathrm{d} a \\
& =\frac{4}{3} \pi \delta_{i} N_{i}(r) A B
\end{aligned}
$$

where $B \equiv \int_{a_{1}}^{a_{2}} a^{3-q} \mathrm{~d} a$ and $\delta_{i}$ is the mass density of the material composing grains of type $i$. But $\rho_{i}(r)$ can also be expressed as $\rho_{i}(r)=f_{i} \rho(r)=f_{i} C_{1} r^{-p}$, where $f_{i}$ is the fractional mass density abundance for grains of chemical type $i$. We can then solve for $N_{i}(r)$ in terms of the constant $C_{1}$ :

$$
\begin{aligned}
N_{i}(r) & =\frac{f_{i} C_{1} r^{-p}}{\frac{4}{3} \pi \delta_{i} A B} \\
& =C_{1} D_{i} r^{-p}
\end{aligned}
$$

where $D_{i} \equiv \frac{f_{i}}{\frac{4}{3} \pi \delta_{i} A B}$, and depends only on the grain properties. Equation (B.2) can then be rewritten in terms of $D_{i}$ and $C_{1}$ :

$$
\begin{aligned}
\tau_{v}= & \int_{R_{\text {in }}}^{R_{\text {out }}} \mathrm{d} r \sum_{i} N_{i}(r) \int_{a_{1}}^{a_{2}} \mathrm{~d} a \pi a^{2} Q_{i, v}(a) \mathcal{F}(a) \\
= & \int_{R_{\text {in }}}^{R_{\text {out }}} \mathrm{d} r C_{1} \sum_{i} D_{i} r^{-p} \\
& \int_{a_{1}}^{a_{2}} \mathrm{~d} a\left(\pi a^{2}\right) Q_{i, v}(a) A a^{-q}
\end{aligned}
$$

\begin{tabular}{|c|c|c|c|c|}
\hline Region & $\lambda(\mu \mathrm{m})$ & Flux (mJy) & Error (mJy) & Ref. \\
\hline $\mathrm{OPT}+\mathrm{NIR}$ & 0.36 & 74.76 & 6.88 & A11 \\
\hline $\mathrm{OPT}+\mathrm{NIR}$ & 0.44 & 129.84 & 11.96 & A11 \\
\hline $\mathrm{OPT}+\mathrm{NIR}$ & 0.44 & 191.17 & 21.12 & A2 \\
\hline $\mathrm{OPT}+\mathrm{NIR}$ & 0.55 & 174.22 & 16.04 & A11 \\
\hline $\mathrm{OPT}+\mathrm{NIR}$ & 0.55 & 242.72 & 26.82 & $\mathrm{~A} 2$ \\
\hline $\mathrm{OPT}+\mathrm{NIR}$ & 0.64 & 273.24 & 25.16 & A11 \\
\hline $\mathrm{OPT}+\mathrm{NIR}$ & 1.2 & 147.27 & 13.56 & $\mathrm{~A} 27\left(48^{\prime \prime}\right)$ \\
\hline $\mathrm{OPT}+\mathrm{NIR}$ & 1.2 & 381.70 & 8.44 & A28 \\
\hline $\mathrm{OPT}+\mathrm{NIR}$ & 1.6 & 163.15 & 15.02 & $\mathrm{~A} 27\left(48^{\prime \prime}\right)$ \\
\hline $\mathrm{OPT}+\mathrm{NIR}$ & 1.6 & 412.86 & 10.64 & A28 \\
\hline $\mathrm{OPT}+\mathrm{NIR}$ & 2.2 & 133.53 & 18.44 & $\mathrm{~A} 27\left(48^{\prime \prime}\right)$ \\
\hline $\mathrm{OPT}+\mathrm{NIR}$ & 2.2 & 329.58 & 12.75 & A28 \\
\hline $\mathrm{OPT}+\mathrm{NIR}$ & 3.8 & 145.01 & 13.35 & $\mathrm{~A} 27\left(7^{\prime \prime} .5\right)$ \\
\hline MIR & 4.5 & 125.0 & 30.0 & A29 \\
\hline MIR & 7.8 & 865.7 & 233.7 & A30 $\left(8^{\prime \prime} \cdot 2\right)$ \\
\hline MIR & 8.3 & 1076.20 & 99.10 & $\mathrm{~A} 27\left(7^{\prime \prime} .5\right)$ \\
\hline MIR & 8.6 & 1153.9 & 115.4 & A30 $\left(8^{\prime \prime} \cdot 2\right)$ \\
\hline MIR & 9.4 & 1215.50 & 111.90 & $\mathrm{~A} 27\left(7^{\prime \prime} .5\right)$ \\
\hline MIR & 9.6 & 1065.9 & 149.2 & A30 $\left(8^{\prime \prime} \cdot 2\right)$ \\
\hline MIR & 10.0 & 1297.7 & 77.9 & A30 $\left(8^{\prime \prime} \cdot 2\right)$ \\
\hline MIR & 10.3 & 1200.0 & 20.0 & $\mathrm{~A} 27\left(7^{\prime \prime} .5\right)$ \\
\hline MIR & 10.3 & 1372.00 & 126.30 & $\mathrm{~A} 27\left(7^{\prime \prime} .5\right)$ \\
\hline MIR & 10.4 & 1539.0 & 169.3 & A30 $\left(8^{\prime \prime} .2\right)$ \\
\hline MIR & 10.8 & 1340.0 & 40.0 & $\mathrm{~A} 4$ \\
\hline MIR & 11.4 & 1573.9 & 188.9 & A30 $\left(8^{\prime \prime} \cdot 2\right)$ \\
\hline MIR & 11.7 & 2200.0 & 140.0 & A31 \\
\hline MIR & 12.0 & 1960.0 & 300.0 & A29 \\
\hline MIR & 12.0 & 2720.0 & 33.0 & $\mathrm{~A} 27\left(7^{\prime \prime} .5\right)$ \\
\hline MIR & 12 & 2215.60 & 204.00 & $\mathrm{~A} 2$ \\
\hline MIR & 12.4 & 2041.8 & 449.2 & A30 ( $\left.8^{\prime \prime} .2\right)$ \\
\hline MIR & 14.9 & 3900.0 & 400.0 & A29 \\
\hline MIR & 17.4 & 7910.20 & 728.40 & $\operatorname{A} 27\left(7^{\prime \prime} .5\right)$ \\
\hline MIR & 18.7 & 2900.0 & 190.0 & A31 \\
\hline MIR & 20.0 & 6098.7 & 914.8 & A30 $\left(8^{\prime \prime} .2\right)$ \\
\hline MIR & 25.0 & 13060.0 & 48.0 & $\mathrm{~A} 2$ \\
\hline FIR-mm & 60 & 30000.0 & 54.0 & A2 \\
\hline FIR-mm & 100 & 30920.0 & 95.0 & A2 \\
\hline FIR-mm & 450 & 2200.0 & $\mathrm{UL}$ & This paper \\
\hline FIR-mm & 850 & 180.0 & 20.0 & This paper \\
\hline FIR-mm & 1200 & 114.0 & 4.0 & A29 \\
\hline Radio & 2.0 & 54.0 & 5.00 & A32 \\
\hline Radio & 3.6 & 58.0 & 10.00 & A33 \\
\hline Radio & 6.0 & 75.0 & 10.00 & $\mathrm{~A} 2$ \\
\hline Radio & 6.0 & 49.0 & 5.00 & A32 \\
\hline Radio & 6.2 & 90.0 & 12.00 & $\mathrm{~A} 2$ \\
\hline Radio & 20.0 & 55.0 & 10.00 & A32 \\
\hline Radio & 21.4 & 90.0 & 40.00 & A32 \\
\hline Radio & 73.5 & 128.0 & 10.00 & A34 \\
\hline$\pi$ & $\begin{array}{l}\frac{R_{\mathrm{out}}^{1-p}-R_{\mathrm{in}}^{1-p}}{1-p} \\
\ln \frac{R_{\mathrm{out}}}{R_{\text {in }}}\end{array}$ & 1 & & \\
\hline
\end{tabular}

Table A6. SED for NGC 5253

Here $\left\langle Q_{i, v}(a)\right\rangle$ is a mean value, since it has been taken out of the integral.

The quantities $R_{\text {in }}, R_{\text {out }}, p, a_{1}, a_{2}, q$ are input; hence we can calculate the constants $A$ and $B$. DUSTY requires the specification of the fractional number abundances of the grains $F_{i}=N_{i} / \sum_{i} N_{i}$, rather than the fractional mass density abundances $f_{i}$. However, the two quantities are related through:

$f_{i}=\rho_{i} / \rho=\frac{\delta_{i} N_{i}}{\sum_{i} \delta_{i} N_{i}}=\frac{\delta_{i} F_{i}}{\sum_{i} \delta_{i} F_{i}}$

Therefore, with the density $\delta_{i}$ for each grain type $i, D_{i}$ can be calculated. Assigning a mean extinction efficiency factor $\left\langle Q_{i}\right\rangle$ to each grain type, we can finally relate $\tau_{v}$ to the 
Table A7. SED for SBS 0335-052

\begin{tabular}{|c|c|c|c|c|}
\hline Region & $\lambda(\mu \mathrm{m})$ & Flux (mJy) & Error (mJy) & Ref. \\
\hline OPT+NIR & 0.36 & 0.58 & 0.02 & A35 \\
\hline $\mathrm{OPT}+\mathrm{NIR}$ & 0.44 & 0.70 & 0.01 & A35 \\
\hline $\mathrm{OPT}+\mathrm{NIR}$ & 0.55 & 0.80 & 0.01 & A36 \\
\hline $\mathrm{OPT}+\mathrm{NIR}$ & 0.64 & 0.73 & 0.01 & A35 \\
\hline $\mathrm{OPT}+\mathrm{NIR}$ & 0.79 & 0.45 & 0.00 & A36 \\
\hline $\mathrm{OPT}+\mathrm{NIR}$ & 1.2 & 0.50 & 0.02 & A37 \\
\hline $\mathrm{OPT}+\mathrm{NIR}$ & 1.6 & 0.37 & 0.02 & A37 \\
\hline $\mathrm{OPT}+\mathrm{NIR}$ & 2.2 & 0.44 & 0.02 & A37 \\
\hline $\mathrm{OPT}+\mathrm{NIR}$ & 3.8 & 0.58 & 0.11 & A 38 \\
\hline MIR & 5.3 & 0.9 & 0.1 & A39 \\
\hline MIR & 6.0 & 2.9 & 0.1 & A39 \\
\hline MIR & 6.7 & 5.5 & 0.4 & A 40 \\
\hline MIR & 7.0 & 5.8 & 0.1 & A39 \\
\hline MIR & 7.7 & 8.9 & 0.5 & $\mathrm{~A} 40$ \\
\hline MIR & 8.1 & 9.8 & 0.1 & A39 \\
\hline MIR & 9.1 & 11.6 & 0.1 & A39 \\
\hline MIR & 9.9 & 11.3 & 0.1 & A39 \\
\hline MIR & 10.2 & 12.0 & 0.1 & A 39 \\
\hline MIR & 10.8 & 10.3 & 5.1 & A 41 \\
\hline MIR & 10.9 & 16.0 & 0.1 & A39 \\
\hline MIR & 11.3 & 20.7 & 0.8 & $\mathrm{~A} 40$ \\
\hline MIR & 11.3 & 18.4 & 0.1 & A39 \\
\hline MIR & 11.8 & 24.0 & 0.1 & A39 \\
\hline MIR & 12.0 & 20.5 & 0.6 & $\mathrm{~A} 40$ \\
\hline MIR & 12.0 & 25.1 & 0.1 & A39 \\
\hline MIR & 13.3 & 37.3 & 0.1 & A39 \\
\hline MIR & 14.3 & 41.7 & 0.1 & A39 \\
\hline MIR & 14.9 & 35.2 & 0.9 & $\mathrm{~A} 40$ \\
\hline MIR & 15.1 & 42.8 & 0.1 & A39 \\
\hline MIR & 17.0 & 45.3 & 0.1 & A39 \\
\hline MIR & 18.0 & 49.3 & 0.1 & A39 \\
\hline MIR & 19.0 & 55.3 & 0.1 & A39 \\
\hline MIR & 20.1 & 55.7 & 0.1 & A39 \\
\hline MIR & 21.0 & 50.2 & 15.1 & A 41 \\
\hline MIR & 21.0 & 57.5 & 0.1 & A39 \\
\hline MIR & 21.6 & 60.1 & 0.1 & A39 \\
\hline MIR & 22.0 & 70.0 & 11.0 & A39 \\
\hline MIR & 22.5 & 59.0 & 0.1 & A39 \\
\hline MIR & 23.1 & 59.0 & 0.1 & A39 \\
\hline MIR & 24.6 & 60.8 & 0.1 & A39 \\
\hline MIR & 25.1 & 61.9 & 0.1 & A39 \\
\hline MIR & 25.9 & 63.0 & 0.1 & A 39 \\
\hline MIR & 26.6 & 64.2 & 0.1 & A39 \\
\hline MIR & 27.4 & 64.2 & 0.1 & A39 \\
\hline MIR & 28.4 & 65.0 & 0.1 & A39 \\
\hline MIR & 29.4 & 64.6 & 0.1 & A39 \\
\hline FIR-mm & 30.2 & 62.6 & 0.1 & A39 \\
\hline FIR-mm & 31.6 & 62.0 & 0.1 & A39 \\
\hline FIR-mm & 65 & 112.0 & 21.0 & A 41 \\
\hline FIR-mm & 65 & 44.0 & 20.0 & This paper \\
\hline FIR-mm & 850 & 5.0 & UL & This paper \\
\hline Radio & 0.130914 & 11.00 & UL & A 42 \\
\hline Radio & 0.267672 & 2.30 & $\mathrm{UL}$ & A 42 \\
\hline Radio & 1.3 & 0.5 & 0.08 & $\mathrm{~A} 43$ \\
\hline Radio & 2.0 & 0.4 & 0.07 & A 43 \\
\hline Radio & 3.5 & 0.7 & 0.04 & A 43 \\
\hline Radio & 6.2 & 0.8 & 0.09 & A 43 \\
\hline Radio & 20.1 & 0.4 & 0.13 & A 42 \\
\hline Radio & 20.5 & 0.5 & 0.06 & A 43 \\
\hline
\end{tabular}

constant $C_{1}$, necessary to derive the dust mass as given in Eq. (B.1). In our calculations, we have assumed the following physical properties for the grains: $\delta_{\mathrm{AMC}}=1.81 \mathrm{~g} \mathrm{~cm}^{-3}$ (Kim et al. 1994); $\delta_{\text {silicate }}=3.3 \mathrm{~g} \mathrm{~cm}^{-3}$ (Draine \& Lee 1984); $\delta_{\text {graphite }}=2.26 \mathrm{~g} \mathrm{~cm}^{-3}$ (Draine \& Lee 1984); and $\left\langle Q_{i}\right\rangle=2$ for all grain types (see Spitzer 1978). 